\title{
Reflections in abstract Coxeter groups
}

\author{
W. N. Franzsen, R. B. Howlett and B. Mühlherr
}

\begin{abstract}
Let $W$ be a Coxeter group and $r \in W$ a reflection. If the group of order 2 generated by $r$ is the intersection of all the maximal finite subgroups of $W$ that contain it, then any isomorphism from $W$ to a Coxeter group $W^{\prime}$ must take $r$ to a reflection in $W^{\prime}$. The aim of this paper is to show how to determine, by inspection of the Coxeter graph, the intersection of the maximal finite subgroups containing $r$. In particular we show that the condition above is satisfied whenever $W$ is infinite and irreducible, and has the property that all rank two parabolic subgroups are finite. So in this case all isomorphisms map reflections to reflections.
\end{abstract}

Mathematics Subject Classification (2000). 20F55; 51F15.

Keywords. Coxeter group, isomorphism problem, automorphism, reflection.

\section{Introduction}

The dihedral group of order 12 can be considered as Coxeter group of type $I_{2}(6)$ or as Coxeter group of type $A_{1} \times I_{2}(3)$. This example shows that, in general, the set of reflections in a Coxeter system is not determined by the abstract group $W$ alone, but does indeed depend on the choice of the Coxeter generating set $R$. However there are a lot of examples of Coxeter systems $(W, R)$ where the abstract group does determine the set of reflections or even the set $R$ up to $W$-conjugacy. The main motivation for the present paper is to show that the latter holds for infinite Coxeter groups having a finite, irreducible and 2-spherical Coxeter generating set, which is our Theorem 1 below.

In view of the main result of [5] it suffices to show that these Coxeter groups determine the set of reflections. In order to achieve this goal we provide a handy criterion for an involution in an abstract Coxeter group $W$ to be a reflection with respect to any Coxeter generating set of $W$. Our principal observation is the following. Let $(W, R)$ be a Coxeter system and let $w \in W$ be an involution. If $w \notin R^{W}$, then the centralizer of $w$ in $W$ contains a finite normal subgroup properly containing $\langle w\rangle$. This is an immediate consequence of Richardson's result in [16]. Thus, if $w \in W$ is an involution having the property that $\langle w\rangle$ is a maximal finite normal subgroup of its 
centralizer in $W$, then $w$ is a reflection with respect to any Coxeter generating set of $W$.

It turns out that it is more convenient to work with the finite continuation of an involution rather than to consider finite normal subgroups of its centralizer. The finite continuation of a finite order element $w$ in a Coxeter group is defined to be the intersection of all maximal finite subgroups containing it; we write $\mathrm{FC}(w)$ for the finite continuation of $w$. In this paper we restrict our attention to finitely generated Coxeter groups. For these it is a consequence of a result of Tits that every element of finite order is contained in some maximal finite subgroup; so $\mathrm{FC}(w)$ is a finite subgroup of $W$ (see Corollary 14 below). The main result of the present paper is a complete description of the finite continuation of a simple reflection in a Coxeter system of finite rank. Its proof constitutes the bulk of this paper.

Main Result. Let $(W, R)$ be a Coxeter system of finite rank. Then the following holds.

a) For each $r \in R$ the finite continuation of $r$ can be described.

b) Given an involution $w \in W$ such that $\mathrm{FC}(w)=\langle w\rangle$, then $w \in R^{W}$.

Part a) of our main result is Theorem 7. Its precise statement requires some preparation. Part b) is Corollary 24.

The main result of this paper is in fact the first of two steps to reduce the isomorphism problem for Coxeter groups to its 'reflection-preserving' version. The second step is given in [12]. We refer to [15] for further information about the applications to the general isomorphism problem.

A special instance of the isomorphism problem for Coxeter groups is the question about their rigidity (see [3] for further information). In combination with the main result of [5] a consequence of our main result is the following rigidity result.

Theorem 1. Let $(W, R)$ be an irreducible, non-spherical Coxeter system such that $R$ is finite and such that $r r^{\prime}$ has finite order for all $r, r^{\prime} \in R$. Then the following assertions hold.

a) For each $r \in R$ we have $\mathrm{FC}(r)=\langle r\rangle$.

b) If $S \subseteq W$ is such that $(W, S)$ is a Coxeter system, then there exists $w \in W$ such that $S^{w}=R$.

c) All automorphisms of $W$ are inner-by-graph.

In the language of [3], Part b) of the previous theorem means that an infinite, irreducible, 2-spherical Coxeter system is strongly rigid. Part c), which is an immediate consequence of Part b), improves the result of [13]. 
To conclude this introduction we remark that characterization results for reflections in even Coxeter groups have been obtained in [1]. Some of the results there can be deduced as corollaries of our main result as well.

Acknowledgements. The authors thank Frédéric Haglund for helpful discussions on the subject, and the Australian Research Council for support. The third author thanks the University of Sydney for hospitality when this research was undertaken.

\section{Precise statement of the main result}

Recall that a Coxeter group is a group with a presentation of the form

$$
\left.W=\operatorname{gp}\left\langle\left\{r_{a} \mid a \in \Pi\right\}\right|\left(r_{a} r_{b}\right)^{m_{a b}}=1 \text { for all } a, b \in \Pi\right\rangle
$$

where $\Pi$ is some indexing set, whose cardinality is called the rank of $W$ (relative to this presentation), and the $m_{a b}$ satisfy the following conditions: $m_{a b}=m_{b a}$, each $m_{a b}$ lies in the set $\{m \in \mathbb{Z} \mid m \geqslant 1\} \cup\{\infty\}$, and $m_{a b}=1$ if and only if $a=b$. When $m_{a b}=\infty$ the relation $\left(r_{a} r_{b}\right)^{m_{a b}}=1$ is interpreted as vacuous. We shall restrict attention to Coxeter groups of finite rank.

A reduced expression for an element $w \in W$ is a minimal length word expressing $w$ as a product of elements of the distinguished generating set $\left\{r_{a} \mid a \in \Pi\right\}$. We define $\ell(w)$ to be the length of a reduced expression for $w$.

As is well known (and as we shall describe in Section 3 below), every Coxeter group $\mathrm{W}$ can be realized geometrically as a group generated by reflections. In this realization of $W$ the reflections in $W$ are the conjugates of the generators $r_{a}$.

The Coxeter graph associated with the presentation above is the graph with vertex set $\Pi$ and edge set consisting of those pairs of vertices $\{a, b\}$ for which $m_{a b} \geqslant 3$. The edge $\{a, b\}$ is given the label $m_{a b}$. The components of $\Pi$ are the connected components of the graph, and we say that $W$ is irreducible if the graph is connected.

For each $I \subseteq \Pi$ we define $W_{I}$ to be the subgroup of $W$ generated by the set $\left\{r_{a} \mid a \in I\right\}$; we call these subgroups the visible subgroups of $W$. A parabolic subgroup of $W$ is any conjugate of a visible subgroup. We say that $I \subseteq \Pi$ is spherical if $W_{I}$ is finite, and we say that $\Pi$ (or $W$ ) is $k$-spherical if all $k$-element subsets of $\Pi$ are spherical.

The definitions given so far are fairly standard. In order to facilitate the precise statement of the main result, we introduce some nonstandard notation and terminology (in Definitions 2, 3, 4, 5 and 6 below).

Definition 2. If $w \in W$ has finite order, define the finite continuation of $w$, written $\mathrm{FC}(w)$, to be the intersection of all maximal finite subgroups of $W$ containing $w$. 
Definition 3. The odd graph of $W$ is the graph $\Omega(\Pi)$ obtained from the Coxeter graph by deleting the edges whose labels are infinite or even. For each $a \in \Pi$ we define $\operatorname{Odd}(a)$ to be the connected component of $\Omega(\Pi)$ containing $a$. For each connected component $M$ of $\Omega(\Pi)$ we define $\mathrm{E}(M)$ to be the union of $M$ with the set of all $b \in \Pi$ such that $m_{c b}$ is even for some $c \in M$. We also abbreviate $\mathrm{E}(\operatorname{Odd}(a))$ to $\operatorname{EOdd}(a)$.

In the discussions below, when we refer to the components of $\mathrm{E}(M)$ we regard $\mathrm{E}(M)$ as the full subgraph of the Coxeter graph spanned by the vertices in $\mathrm{E}(M)$. In other words, the edges with even and infinite labels, deleted when forming the odd graph, are restored in $\mathrm{E}(M)$.

Note that if $a \in L \subseteq \Pi$ and $W_{L}$ is finite then $m_{a b}<\infty$ for all $b \in L$. Whether $m_{a b}$ is odd or even it follows that $b \in \operatorname{EOdd}(a)$. Thus $L \subseteq \operatorname{EOdd}(a)$.

Definition 4. Let $M \subseteq \Pi$ be a connected component of $\Omega(\Pi)$. We call $b \in \Pi \backslash M$ a $C_{3}$-neighbour of $M$ if $m_{b c} \in\{2,4\}$ for all $c \in E(M)$, the case $m_{b c}=4$ occurring for at least one $c$, and for each $c \in E(M)$ with $m_{b c}=4$ there is an $a \in M$ such that the following conditions are satisfied:

(1) $m_{b a}=2$ and $m_{c a}=3$, and $m_{c d}=\infty$ for all $d \in M \backslash\{a, c\}$;

(2) for all $e \in \Pi \backslash(M \cup\{b\})$, either $m_{c e}=\infty$ or $m_{a e}=m_{c e}=m_{b e}=2$.

Definition 5. Let $M \subseteq \Pi$ be a connected component of $\Omega(\Pi)$, and let $a \in M$ and $b \in \Pi \backslash M$. We call the pair $(a, b)$ a focus of $M$ in $\Pi$ if the following conditions all hold.

(1) All the edge labels of $M$ are 3, and $M$ is a tree.

(2) For each $c \in M$, the set $C[b . . c] \subseteq \Pi$ consisting of $b$ and those elements of $M$ that form the path from $a$ to $c$ in $M$ constitutes a system of type $C_{k}$ (for some $k$ dependent on $c$ ).

(3) If $c, d \in M \cup\{b\}$ with $c \notin C[b . . d]$ and $d \notin C[b . . c]$ then $m_{c d}=\infty$.

(4) If $m_{c e} \neq \infty$ for some $c \in M$ and $e \in \Pi \backslash(M \cup\{b\})$, then $m_{c e}=2=m_{d e}$ for all $d \in C[b . . c]$.

(5) The vertices of $M \cup\{b\}$ do not form a spherical component of $\mathrm{E}(M)$.

Definition 6. Let $M \subseteq \Pi$ be a connected component of $\Omega(\Pi)$, and let $a, b \in M$. We call the two-element set $\{a, b\}$ a half focus of $M$ in $\Pi$ if $m_{a b}=2$ and the following conditions all hold.

(1) We have $m_{a c}=m_{b c} \in\{2,3\}$ for all $c \in M \backslash\{a, b\}$, and $m_{a c}=m_{b c} \in\{2, \infty\}$ for all $c \in \Pi \backslash M$.

(2) All the edge labels of $M \backslash\{b\}$ are 3, and $M \backslash\{b\}$ is a tree. 
(3) For each $c \in M \backslash\{a, b\}$, the set $D[a, b . . c] \subseteq \Pi$ consisting of $b$ and those elements of $M \backslash\{b\}$ that form the path from $a$ to $c$ constitutes a system of type $D_{k}$ (for some $k$ dependent on $c$ ).

(4) If $c, d \in M \backslash\{a, b\}$ with $c \notin D[a, b . . d]$ and $d \notin D[a, b . . c]$ then $m_{c d}=\infty$.

(5) If $m_{c e} \neq \infty$ for some $c \in M \backslash\{a, b\}$ and $e \in \Pi \backslash M$, then $m_{c e}=2=m_{d e}$ for all $d \in D[a, b . . c]$.

(6) The vertices of $M$ do not form a spherical component of $\mathrm{E}(M)$.

We are now able to give a precise statement of Part a) of our main result

Theorem 7. For each connected component $M$ of $\Omega(\Pi)$ there is at least one $a \in M$ such that $\mathrm{FC}\left(r_{a}\right)$ is a visible subgroup of $W$. We have the following possibilities.

Case A: Suppose that the component of $\mathrm{E}(M)$ containing $M$ is spherical, and let $a \in M$ be arbitrary. Then $\mathrm{FC}\left(r_{a}\right)=W_{J}$, where $J$ is the union of the spherical components of $\mathrm{E}(M)$.

Case B: Suppose that the component of $\mathrm{E}(M)$ containing $M$ is not spherical, and $M$ does not have any focus or half-focus in $\Pi$, and let $J^{\prime}$ be the union of the spherical components of $\mathrm{E}(M)$ and the set of $C_{3}$-neighbours of $M$. If $a \in M$ is not adjacent in $\Pi$ to any $C_{3}$-neighbour of $M$ then $\mathrm{FC}\left(r_{a}\right)=W_{J^{\prime} \cup\{a\}}$, and if $a \in M$ is adjacent in $\Pi$ to a $C_{3}$-neighbour of $M$ then $\mathrm{FC}\left(r_{a}\right)$ is not visible.

Case C: Suppose that $(a, b)$ is a focus of $M$. Then $\mathrm{FC}\left(r_{a}\right)=W_{J}$ where $J$ is the union of $\{a, b\}$ and the spherical components of $\mathrm{E}(M)$, and $\mathrm{FC}\left(r_{c}\right)$ is not visible for any $c \in M \backslash\{a\}$.

Case D: Suppose that $\{a, b\}$ is a half-focus of $M$. Then $\mathrm{FC}\left(r_{a}\right)=\mathrm{FC}\left(r_{b}\right)=W_{J}$, where $J$ is the union of $\{a, b\}$ and the spherical components of $\mathrm{E}(M)$, and $\mathrm{FC}\left(r_{c}\right)$ is not visible for any $c \in M \backslash\{a, b\}$.

\section{Reflections and root systems}

Let $\mathbb{R}$ be the real field, and $V$ the vector space over $\mathbb{R}$ with basis $\Pi$. Let $B$ the bilinear form on $V$ such that for all $a, b \in \Pi$,

$$
B(a, b)=-\cos \left(\pi / m_{a b}\right) .
$$

To make our notation more compact we define $u \cdot v=B(u, v)$ for all $u, v \in V$. Note that $a \cdot a=1$ for all $a \in \Pi$, since $m_{a a}=1$.

For each $a \in V$ such that $a \cdot a=1$, the reflection along $a$ is the transformation of $V$ given by $v \mapsto v-2(a \cdot v) a$. It is well known (see, for example, Corollary 5.4 of [14]) that $W$ has a faithful representation on $V$ such that, for all $a \in \Pi$, the 
element $r_{a}$ acts as the reflection along $a$. We shall identify elements of $W$ with their images in this representation. We also use the notation $r_{a}$ for the reflection along $a$ whenever $a \in V$ satisfies $a \cdot a=1$. It is straightforward to show that each reflection $r_{a}$ preserves the form $B$; hence all elements of $W$ preserve $B$. Furthermore, the equation $g r_{a} g^{-1}=r_{g a}$ holds for all $a \in V$ such that $a \cdot a=1$ and all transformations $g$ that preserve $B$.

We write $\operatorname{Ref}(W)$ for the set of all reflections in $W$. It is immediate from the above comments that if $\Phi=\{w a \mid w \in W, a \in \Pi\}$ then $\left\{r_{b} \mid b \in \Phi\right\} \subseteq \operatorname{Ref}(W)$.

The set $\Phi$ is called the root system of $W$, and elements of $\Phi$ are called roots. Elements of the basis $\Pi$ are called simple roots, and the reflections $r_{a}$ for $a \in \Pi$ are called simple reflections. A root is said to be positive if it has the form $\sum_{a \in \Pi} \lambda_{a} a$ with $\lambda_{a} \geqslant 0$ for all $a \in \Pi$, and negative otherwise. We write $\Phi^{+}$for the set of all positive roots and $\Phi^{-}$for the set of all negative roots.

Lemma 8. With the notation as above, the following statements hold.

(1) Every negative root has the form $\sum_{a \in \Pi} \lambda_{a} a$ with $\lambda_{a} \leqslant 0$ for all $a \in \Pi$. Furthermore, $\Phi^{-}=\left\{-b \mid b \in \Phi^{+}\right\}$.

(2) If $w \in W$ and $a \in \Pi$ then

$$
\ell\left(w r_{a}\right)= \begin{cases}\ell(w)+1 & \text { if } w a \in \Phi^{+}, \\ \ell(w)-1 & \text { if } w a \in \Phi^{-} .\end{cases}
$$

(3) If $t \in \operatorname{Ref}(W)$ then $t=r_{b}$ for some $b \in \Phi$.

(4) The group $W$ is finite if and only if the bilinear form $B$ is positive definite.

(5) The root system $\Phi$ is finite if and only if the group $W$ is finite.

Proof. Proofs of (1) and (2) can be found in [14, Section 5.4], Theorem 4.1 in [7] includes both (4) and (5), and (3) is [13, Lemma 2.2].

The following result is well known.

Lemma 9. Let $a \in \Pi$. Then $\operatorname{Odd}(a)=\Pi \cap W a$.

For each $w \in W$ we define $N(w)=\left\{b \in \Phi^{+} \mid w b \in \Phi^{-}\right\}$. By Part (2) of Lemma 8 , if $w \neq 1$ then $N(w) \cap \Pi \neq \emptyset$. An easy induction shows that $N(w)$ has exactly $\ell(w)$ elements. In particular, $N(w)$ is a finite set. It is also easily shown that if $\Phi$ is finite then there is a unique $w \in W$ such that $N(w)=\Phi^{+}$. This element, which we denote by $w_{\Pi}$, is also the unique element of maximal length in $W$ (which is a finite group). Furthermore, $w_{\Pi} \Pi=-\Pi$. 
For each $\Gamma \subseteq \Phi$ the subgroup $W_{\Gamma}$ generated by the set $\left\{r_{a} \mid a \in \Gamma\right\}$ is called a reflection subgroup of $W$. The set $\Phi_{\Gamma}=\left\{a \in \Phi \mid r_{a} \in W_{\Gamma}\right\}$ is called the root subsystem generated by $\Gamma$. Let $\Phi_{\Gamma}^{+}=\Phi_{\Gamma} \cap \Phi^{+}$and $\Phi_{\Gamma}^{-}=\Phi_{\Gamma} \cap \Phi^{-}$, and define

$$
\Pi_{\Gamma}=\left\{a \in \Phi_{\Gamma}^{+} \mid N\left(r_{a}\right) \cap \Phi_{\Gamma}=\{a\}\right\} .
$$

The main theorem of Deodhar [8] and Theorem (3.3) of Dyer [9] yield the following result.

Theorem 10. For each $\Gamma \subseteq \Phi$ the group $W_{\Gamma}$ is a Coxeter group on the generating set $\left\{r_{a} \mid a \in \Pi_{\Gamma}\right\}$. The set $\left\{a \cdot b \mid a, b \in \Pi_{\Gamma}\right.$ and $\left.a \neq b\right\}$ is a subset of $\mathscr{C}=$ $\{-\cos (\pi / m) \mid 2 \leqslant m \in \mathbb{Z}\} \cup(-\infty,-1]$. Moreover, if $\Delta$ is any subset of $\Phi^{+}$such that $\{a \cdot b \mid a, b \in \Delta$ and $a \neq b\} \subseteq \mathscr{C}$ then $W_{\Delta}$ is a Coxeter group on the generating $\operatorname{set}\left\{r_{a} \mid a \in \Delta\right\}$.

Note that the notation $W_{\Gamma}$ introduced above is an extension of the notation for visible subgroups introduced in Section 2. However, if $\Gamma \nsubseteq \Pi$ then $W_{\Gamma}$ need not be visible.

It is clear that if $I \subseteq \Pi$ then $W_{I}$ preserves the subspace $V_{I}$ of $V$ spanned by $I$, and acts on this subspace as a Coxeter group with $I$ as its set of simple roots. In this case $\Phi_{I}=\Phi \cap V_{I}$ and $\Pi_{I}=I$.

The following simple facts are well known.

Lemma 11. In the above situation, $\Phi_{I}=\Phi \cap V_{I}$. Furthermore, $w \in W$ normalizes $W_{I}$ if and only if $w \Phi_{I}=\Phi_{I}$. In particular, for all $a \in \Phi$, the reflection $r_{a}$ normalizes $W_{I}$ if and only if $a \in \Phi_{I}$ or $a \cdot b=0$ for all $b \in I$.

Suppose that $I \subseteq \Pi$ and $a \in \Pi \backslash I$, and let $L$ be the component of (the Coxeter graph of) $I \cup\{a\}$ to which $a$ belongs. If $W_{L}$ is finite we define $v[a, I]=w_{L} w_{L \backslash\{a\}}$. It is easily seen that $v[a, I] I \subseteq I \cup\{a\}$, and that $v[a, I] b=b$ for all $b \in I \backslash L$. In particular, $v[a, I] I \in \mathscr{I}=\{J \subseteq \Pi \mid J=w I$ for some $w \in W\}$. It was proved in [11] (for finite Coxeter groups) and in [7] (in the general case) that every element $w \in W$ satisfying $w I \subseteq \Pi$ can be expressed as a product of elements of the form $v\left[a, I^{\prime}\right]$, with $I^{\prime} \in \mathscr{I}$ and $a \in \Pi \backslash I^{\prime}$. That is,

$$
w=v\left[a_{1}, I_{1}\right] v\left[a_{2}, I_{2}\right] \ldots v\left[a_{n}, I_{n}\right]
$$

for some $I_{i}, a_{i}$ such that (for each $i$ ) the component of $I_{i} \cup\left\{a_{i}\right\}$ containing $a_{i}$ corresponds to a finite visible subgroup, $v\left[a_{i}, I_{i}\right] I_{i}=I_{i-1}$ for $1<i \leqslant n$, and $I_{n}=I$. Furthermore, the following result holds.

Proposition 12. Let $I, J \subseteq \Pi$. Then $\left\{w \in W \mid w W_{I} w^{-1}=W_{J}\right\}=N(J, I) W_{I}$, where $N(J, I)=\{w \in W \mid w I=J\}$. Furthermore, for each $w \in N(J, I)$ and each $a \in \Pi \cap N(w)$ there is an expression for $w$ of the form (3.1) above, with $\left(a_{n}, I_{n}\right)=(a, I)$ and $\ell(w)=\sum_{i=1}^{n} \ell\left(v\left[a_{i}, I_{i}\right]\right)$. 
The following lemma, which appears in [2, Exercise 2d, p. 130], is fundamental to all of our arguments.

Lemma 13 (Tits). If $W$ is a Coxeter group and $H \leqslant W$ is finite, then $H$ is contained in a finite parabolic subgroup of $W$.

One immediate consequence of Lemma 13 is that every maximal finite subgroup of a Coxeter group is parabolic. Another consequence of the previous lemma is that each finite subgroup of $W$ is contained in a maximal finite parabolic subgroup. (Remember that we always assume that $W$ is finitely generated.) Thus the set of maximal finite subgroups of $W$ containing a given finite order element of $W$ is not empty, and hence we have the following fact.

Corollary 14. If $w \in W$ has finite order, then $\mathrm{FC}(w)$ is a well-defined finite subgroup of $W$.

Lemma 15 (Kilmoyer). Let $I, J \subseteq \Pi$. Then every $\left(W_{I}, W_{J}\right)$ double coset in $W$ contains a unique element of minimal length; moreover, if $d$ is the minimal length element of $W_{I} d W_{J}$ then $W_{I} \cap d W_{J} d^{-1}=W_{K}$, where $K=I \cap d J$.

Proof. See [6, Theorem 2.7.4].

Corollary 16. The intersection of a finite number of parabolic subgroups is a parabolic subgroup.

The following consequence of Lemmas 13 and 15 is proved in [10, Lemma 11].

Lemma 17. If $J$ is a maximal spherical subset of $\Pi$ then $W_{J}$ is a maximal finite subgroup of $W$. Furthermore, $W_{J}$ is not conjugate to any other visible subgroup of $W$.

Another important tool in our analysis of automorphisms is the classification of involutions in Coxeter groups, due to Richardson [16].

Proposition 18. Suppose that $w \in W$ is an involution. Then there is a $t \in W$ and a spherical $I \subseteq \Pi$ such that $w=t^{-1} w_{I} t$ with $\ell(w)=\ell\left(w_{I}\right)+2 \ell(t)$, and $w_{I}$ is central in $W_{I}$.

Proof. See [10, Proposition 5].

Definition 19. We say that $I \subseteq \Pi$ is of $(-1)$-type if $W_{I}$ is finite and $w_{I}$ is central in $W_{I}$. 
The reason for the terminology is that $I$ is of $(-1)$-type if and only if there is an element of $W_{I}$ that acts on $V_{I}$ as multiplication by -1 .

We need the following lemma.

Lemma 20. Suppose that $I, J \subset \Pi$ with $I$ of $(-1)$-type, and suppose that $t \in W$ has the property that $t w_{I} t^{-1} \in W_{J}$. Then $t W_{I} t^{-1} \subseteq W_{J}$.

Proof. Let $a \in I$. Then $w_{I}(a)=-a$, and so $\left(t w_{I} t^{-1}\right)(t a)=-t a$, whence it follows that either $t a$ or $-t a$ is in the set $N\left(t w_{I} t^{-1}\right)$. But $N\left(t w_{I} t^{-1}\right) \subseteq \Phi_{J}$; so $t a \in \Phi_{J}$, and therefore $t r_{a} t^{-1}=r_{t a} \in W_{J}$. Since $W_{I}$ is generated by $\left\{r_{a} \mid a \in I\right\}$, the result follows.

In particular, it follows from Lemma 20 that if $I, J$ are both of (-1)-type and $t w_{I} t^{-1}=w_{J}$ then $t W_{I} t^{-1}=W_{J}$. Conversely, suppose that $t W_{I} t^{-1}=W_{J}$, so that in fact $d W_{I} d^{-1}=W_{J}$ for all $d$ in $W_{J} t W_{I}$ (which equals $t W_{I}$ ). Taking $d$ to be the shortest element in $t W_{I}$, Lemma 15 yields that $d I=J$, and hence $x \mapsto d x d^{-1}$ is a length-preserving isomorphism $W_{I} \rightarrow W_{J}$; consequently $d w_{I} d^{-1}=w_{J}$. If $w_{I}, w_{J}$ are central in $W_{I}, W_{J}$ we deduce that $t w_{I} t^{-1}=w_{J}$. So we have proved the following result.

Lemma 21. Suppose that $I, J$ are subsets of $\Pi$ that are both of $(-1)$-type. Then $\left\{t \in W \mid t w_{I} t^{-1}=w_{J}\right\}=\left\{t \in W \mid t W_{I} t^{-1}=W_{J}\right\}$.

Proposition 22. Let $I \subset \Pi$ be of $(-1)$-type. Then $W_{I} \subseteq \mathrm{FC}\left(w_{I}\right)$.

Proof. Let $F$ be a maximal finite subgroup of $W$ such that $w_{I} \in F$. By Lemma 13 there exist $t \in W$ and $J \subseteq \Pi$ such that $t F t^{-1}=W_{J}$. By Lemma 20 and the fact that $w_{I} \in F$ it follows that $t \bar{W}_{I} t^{-1} \subseteq W_{J}$. Hence $W_{I} \subseteq t^{-1} W_{J} t=F$.

Proposition 23. Let $W, W^{\prime}$ be Coxeter groups of finite rank and $\alpha: W \rightarrow W^{\prime}$ an isomorphism. Let $\Pi$ be the set of simple roots corresponding to the distinguished generating set of $W$, and let $a \in \Pi$. If $r_{a}^{\alpha}$ is not a reflection in $W^{\prime}$ then the intersection of all maximal finite subgroups of $W$ containing $r_{a}$ is a parabolic subgroup of order greater than 2.

Proof. Write $\Pi^{\prime}$ for the set of simple roots of $W^{\prime}$. Observe that Lemma 13 and Corollary 16 trivially imply that $\mathrm{FC}\left(r_{a}\right)$ is a parabolic subgroup of $W$.

Since $r_{a}^{\alpha}$ is not a reflection it follows from Proposition 18 that $r_{a}^{\alpha}=t w_{I} t^{-1}$ for some $t \in W^{\prime}$ and $I \subseteq \Pi^{\prime}$ of $(-1)$-type and of rank at least 2. Clearly $\operatorname{FC}\left(r_{a}\right)^{\alpha}=$ $t \mathrm{FC}\left(w_{I}\right) t^{-1}$, and by Proposition 22 we know that $W_{I} \subseteq \mathrm{FC}\left(w_{I}\right)$. Therefore $\left(t W_{I} t^{-1}\right)^{\alpha^{-1}} \subseteq \mathrm{FC}\left(r_{a}\right)$, so that $\mathrm{FC}\left(r_{a}\right)$ has order greater than 2 , as required.

Corollary 24. Let $w \in W$ be an involution such that $\mathrm{FC}(w)=\langle w\rangle$ and let $S \subseteq W$ be such that $(W, S)$ is a Coxeter system. Then $w \in S^{W}$. 


\section{The finite continuation of a reflection}

Let $r$ be a reflection in $W$. Replacing $r$ by $w r w^{-1}$ replaces $\mathrm{FC}(r)$ by $w \mathrm{FC}(r) w^{-1}$, and so choosing $w$ suitably enables us to assume that $\mathrm{FC}(r)=W_{J}$, a visible parabolic subgroup. Furthermore, replacing $r$ by $t r t^{-1}$ for suitable $t \in W_{J}$ enables us to assume that $r=r_{a}$ for some $a \in J$. (Note that these observations yield the first assertion of Theorem 7.)

Proposition 25. Let $a \in J \subseteq \Pi$, and suppose that $W_{J}$ is the intersection of all maximal finite subgroups of $W$ containing $r_{a}$. Then $\left\{w \in W \mid w r_{a} w^{-1} \in W_{J}\right\}$ is a subset of the normalizer of $W_{J}$ in $W$. Thus each $W$-conjugate of $r_{a}$ in $W_{J}$ is $N_{W}\left(W_{J}\right)$-conjugate to $r_{a}$, and $C_{W}\left(r_{a}\right) \subseteq N_{W}\left(W_{J}\right)$. Moreover, if $b \in \Pi \backslash J$ is such that $W_{J \cup\{b\}}$ is infinite then $m_{b c}=\infty$ for all $c \in J$ such that $r_{c}$ is conjugate to $r_{a}$ in $W$.

Proof. Let $\mathscr{S}$ be the set of all maximal finite subgroups of $W$ containing $r_{a}$, so that $W_{J}=\mathrm{FC}\left(r_{a}\right)=\bigcap_{F \in \mathscr{S}} F$. Suppose that $w \in W$ satisfies $w r_{a} w^{-1} \in W_{J}$, and let $F \in \mathscr{S}$. Then $w r_{a} w^{-1} \in W_{J} \subseteq F$, and so $r_{a} \in w^{-1} F w$. Thus $w^{-1} F w$ is a maximal finite subgroup of $W$ containing $r_{a}$, whence $w^{-1} F w \in \mathscr{S}$. So

$$
\bigcap_{F \in \mathscr{S}} F \subseteq \bigcap_{F \in \mathscr{S}} w^{-1} F w
$$

and so $W_{J} \subseteq w^{-1} W_{J} w$. Since $W_{J}$ is finite it follows that $w \in N_{W}\left(W_{J}\right)$.

Suppose that $c \in J$ with $r_{c}=w r_{a} w^{-1}$ for some $w \in W$. Clearly $F \mapsto w F w^{-1}$ is a bijection from the set of maximal finite subgroups of $W$ containing $r_{a}$ to the set of maximal finite subgroups of $W$ containing $r_{c}$, and so $\mathrm{FC}\left(r_{c}\right)=w \mathrm{FC}\left(r_{a}\right) w^{-1}$. But $w \mathrm{FC}\left(r_{a}\right) w^{-1}=w W_{J} w^{-1}=W_{J}$ by the first part of the proof, and so $\operatorname{FC}\left(r_{c}\right)=W_{J}$. Now suppose that $b \in \Pi \backslash J$ with $m_{c b}<\infty$. Then $W_{\{c, b\}}$ is finite, and so contained in a maximal finite subgroup $F$. Since $r_{c} \in F$ we must have $\mathrm{FC}\left(r_{c}\right) \subseteq F$. It follows that the finite group $F$ contains both $W_{J}$ and $r_{b}$, and therefore $W_{J \cup\{b\}}$ is finite.

Assume, as in Proposition 25, that $a \in J \subseteq \Pi$ and $W_{J}=\mathrm{FC}\left(r_{a}\right)$, and suppose now that $J \neq\{a\}$. Suppose that $L \subseteq \Pi$ is such that $J \subseteq L$ and $W_{L}$ is finite. Then $W_{L}$ is a finite Coxeter group possessing a visible parabolic subgroup $W_{J}$ of rank greater than 1 that is normalized by the centralizer of some simple reflection $r_{a} \in W_{J}$. Indeed, $W_{J}$ is normalized by all $w \in W_{L}$ such that $w r_{a} w^{-1} \in W_{J}$. Equivalently, by Lemma 8(3), $\left\{w \in W_{L} \mid w a \in \Phi_{J}\right\} \subseteq N_{W}\left(W_{J}\right)$. This is a very restrictive condition, which we now proceed to examine with a case-by-case investigation of the different types of finite Coxeter groups. For the course of this investigation, we can (and shall) assume that $L=\Pi$.

So we assume for now that $W$ is a finite Coxeter group of rank $n$, and our aim is to find all examples of the following phenomenon: there exist $\{a\} \varsubsetneqq J \subseteq \Pi$ such that 
the set $Q=\left\{w \in W \mid w a \in \Phi_{J}\right\}$ is a subset of $N_{W}\left(W_{J}\right)$. We assume that $J \neq \Pi$, since the condition is trivially satisfied otherwise.

If $K \subseteq \Pi$ is a component of the Coxeter graph such that $J \cap K=\emptyset$ then $W_{K}$ is a direct factor of $N_{W}\left(W_{J}\right)$; moreover, $Q=\left(Q \cap W_{\Pi \backslash K}\right) W_{K}$. So removing $K$ from the graph will have no bearing on whether or not the condition $Q \subseteq N_{W}\left(W_{J}\right)$ holds. So we assume that there are no such components of $\Pi$. Exactly the same comments apply for a component $K$ of $\Pi$ such that $K \subseteq J$. So we also assume that there are none of these.

Assume that $\{a\} \varsubsetneqq J \varsubsetneqq \Pi$ and $Q \subseteq N_{W}\left(W_{J}\right)$. Suppose that $K \subseteq \Pi$ is a component of the Coxeter graph such that $a \notin K$. Then $r_{b} a=a$ for all $b \in K$; so $r_{b} \in Q \subseteq N_{W}\left(W_{J}\right)$, and it follows that $r_{b} c \in \Phi_{J}$ whenever $c \in J$. If $b \cdot c \neq 0$ then $b$ is in the support of $r_{b} c$, and so $r_{b} c \in \Phi_{J}$ implies $b \in J$. Since $K$ is connected it follows that if $K$ contains any element of $J$ then $K \subseteq J$. So either $K \cap J=\emptyset$ or $K \subseteq J$. But we have assumed that there are no such components. So the component of $\Pi$ that contains $a$ is the only component; that is, $\Pi$ is irreducible.

Observe that the group $\operatorname{Stab}(a)=\{w \in W \mid w a=a\}$ is a subset of $Q$ and hence of $N_{W}\left(W_{J}\right)$. Note also that $N_{W}\left(W_{J}\right)=\left\{w \in W \mid w \Phi_{J}=\Phi_{J}\right\}$, which is also the stabilizer of the subspace $V_{J}$ (since $V_{J}$ is the subspace spanned by $\Phi_{J}$ and $\left.\Phi_{J}=V_{J} \cap \Phi\right)$. Now $\operatorname{Stab}(a)$ is a parabolic subgroup of $W$ whose root system is $\Phi \cap a^{\perp}$, and the following table gives the type of this root system in all cases.

\begin{tabular}{c|c}
$W$ & $\operatorname{Stab}(a)$ \\
\hline$A_{n}$ & $A_{n-2}$ \\
$C_{n}$ & $C_{n-2}+A_{1}$ \\
$C_{n}$ & $C_{n-1}$ \\
$D_{n}$ & $D_{n-2}+A_{1}$ \\
$F_{4}$ & $C_{3}$ \\
$E_{6}$ & $A_{5}$
\end{tabular}

\begin{tabular}{c|c}
$W$ & $\operatorname{Stab}(a)$ \\
\hline$E_{7}$ & $D_{6}$ \\
$E_{8}$ & $E_{7}$ \\
$H_{3}$ & $A_{1}+A_{1}$ \\
$H_{4}$ & $H_{3}$ \\
$I_{2}(2 k)$ & $A_{1}$ \\
$I_{2}(2 k+1)$ & $\emptyset$
\end{tabular}

(For $C_{n}$ there are two $W$ orbits of roots, giving two possibilities for $\operatorname{Stab}(a)$. For $F_{4}$ and $I_{2}(2 k)$ there are also two $W$-orbits of roots, but $\operatorname{Stab}(a)$ has the same type of root system whichever orbit $a$ belongs to.) Since each irreducible constituent of its root system spans an irreducible $\operatorname{Stab}(a)$-submodule of $V$, the table shows that as a $\operatorname{Stab}(a)$-module, $V$ has composition length two or three or (in one case only) four: $a$ itself spans a trivial $\operatorname{Stab}(a)$-submodule of dimension 1 , and $a^{\perp}$ is either irreducible of dimension $n-1$ (for types $F_{4}, E_{6}, E_{7}, E_{8}, H_{4}, I_{2}(2 k)$ and one of the $C_{n}$ possibilities), or the direct sum of irreducibles of dimensions 1 and $n-2$ (for types $A_{n}, C_{n}, D_{n}$ when $n>4, H_{3}$ and $I_{2}(2 k+1)$ ), or the direct sum of three irreducibles of dimension 1 (for type $D_{4}$ ). Furthermore, the summands of $a^{\perp}$ are pairwise nonisomorphic as $\operatorname{Stab}(a)$-modules, since even if they are of the same type their centralizers in $\operatorname{Stab}(a)$ are different. 
Since $\{a\} \varsubsetneqq J \varsubsetneqq \Pi$ and $V_{J}$ is $\operatorname{Stab}(a)$-invariant, we see that $a^{\perp}=\left(V_{J} \cap a^{\perp}\right) \oplus V_{J}^{\perp}$, with both summands nonzero $\operatorname{Stab}(a)$-modules. So $\Pi$ is of type $A_{n}, C_{n}, D_{n}$ or $H_{3}$. Furthermore, except in type $D_{4}$, the two direct summands of $a^{\perp}$ are irreducible and not isomorphic, and are therefore the only proper $\operatorname{Stab}(a)$-submodules of $a^{\perp}$. We conclude that $V_{J}$ is spanned by $a$ and one of the summands of $a^{\perp}$, while $V_{J}^{\perp}$ is the other summand. In type $D_{4}$ we similarly deduce that $V_{J}$ is spanned by $a$ and one or two of the three 1-dimensional summands of $a^{\perp}$, and, correspondingly, $V_{J}^{\perp}$ is of either of type $A_{1}+A_{1}$ or of type $A_{1}$.

If $\Pi$ is of type $A_{n}$ then one of the summands of $a^{\perp}$ is of type $A_{n-2}$ while the other is a trivial 1-dimensional $\operatorname{Stab}(a)$-module. If $V_{J}^{\perp}$ is of type $A_{n-2}$ then $V_{J}$ must be of type $A_{1}$, since the orthogonal complement of a subsystem of type $A_{n-2}$ in $A_{n}$ contains only a rank 1 root system. This contradicts the assumption that $\{a\} \varsubsetneqq J$. So $J$ is of type $A_{1}+A_{n-2}$. Since $W_{J}$ is visible, we deduce that $a$ is an end node of the $A_{n}$ diagram, and the node adjacent to $a$ is the unique simple root not in $J$. However, if $n>3$ then the maximal length element of $W$ is in $Q$ but not in the normalizer of $W_{J}$. So $n=3$ and $J=\{a, c\}$, where $c$ is the other end node. It is readily checked that $Q$ has 8 elements and coincides with $N_{W}\left(W_{J}\right)$ (which is generated by $W_{J}$ and an element that interchanges $a$ and $c$ ).

If $\Pi$ is of type $C_{n}$ then one summand of $a^{\perp}$ is of type $C_{n-2}$ and the other of type $A_{1}$. The roots in the $A_{1}$ summand are in the same $W$-orbit as $a$. If $V_{J}^{\perp}$ is the $A_{1}$ component of $a^{\perp}$ then $V_{J}=\left(V_{J}^{\perp}\right)^{\perp}$ is of type $C_{n-2}+A_{1}$. This determines $J$ uniquely, since $W_{J}$ is visible. If $n \geqslant 4$ and $w$ is the longest element of the visible parabolic subgroup of type $A_{n-1}$, then $-w a \in \Pi \backslash\{b\}=J$, but $w \notin N_{W}\left(W_{J}\right)$. This contradicts the fact that $Q \subseteq N_{W}\left(W_{J}\right)$. So $n=3$, and the elements of $J$ are the end nodes $a, c$ of the $C_{3}$ diagram, the middle node $b$ being in the same $W$-orbit as $a$. Since $\Phi_{J}=\{ \pm a, \pm c\}$ and $c$ is not in the same $W$-orbit as $a$ and $b$ we deduce that $Q=\{w \in W \mid w a= \pm a\}$. Furthermore, of the 6 roots in the $W$-orbit of $c$, only $c$ and $-c$ are orthogonal to $a$. So if $w a= \pm a$ then $w c= \pm c$. Thus if $w \in Q$ then $w \Phi_{J}=\Phi_{J}$, as required.

Continuing the discussion of $C_{n}$, suppose now that $V_{J}^{\perp}$ is the $C_{n-2}$ component of $a^{\perp}$. Then $V_{J}=\left(V_{J}^{\perp}\right)^{\perp}$ is of type $C_{2}$. Writing $J=\{a, b\}$, the fact that $\operatorname{Stab}(a)$ is of type $A_{1}+C_{n-2}$ means that it is $b$ rather than $a$ that is the end node of the $C_{n}$ diagram. If we put $c=r_{b} a$ then $\{ \pm c\}$ is the component of $\Phi \cap a^{\perp}$ of type $A_{1}$. It follows that $\{ \pm a\}=\left\{ \pm r_{b} c\right\}$ is the $A_{1}$-component of $\Phi \cap\left(r_{b} a\right)^{\perp}=\Phi \cap c^{\perp}$. We see that $\operatorname{Stab}(a)=\left\langle r_{c}\right\rangle \times W^{\prime}$ and $\operatorname{Stab}(c)=\left\langle r_{a}\right\rangle \times W^{\prime}$, where $W^{\prime}$ is a parabolic (not visible) subgroup of $W$ of type $C_{n-2}$. Indeed, the root system of $W^{\prime}$ is $\Phi \cap V_{J}^{\perp}$. The roots in $\Phi_{J}$ that are in the same $W$-orbit as $a$ are $\pm a$ and $\pm c$, and so

$$
Q=\left\{1, r_{a}, r_{b}, r_{b} r_{a}\right\} \operatorname{Stab}(a)=\left\{1, r_{a}, r_{b}, r_{b} r_{a}\right\}\left\{1, r_{c}\right\} W^{\prime}=W_{J} W^{\prime} .
$$

Hence our requirement that $Q$ stabilizes $\Phi_{J}=\{ \pm a, \pm b\}$ is indeed satisfied. 
If $\Pi$ is of type $D_{n}$ with $n>4$ then one summand of $a^{\perp}$ is of type $D_{n-2}$ and the other of type $A_{1}$. The roots orthogonal to a $D_{n-2}$ subsystem form a system of type $A_{1}+A_{1}$. There are in fact two $W$-orbits of parabolic $A_{1}+A_{1}$ subsystems, and the orthogonal complement of a $D_{n-2}$ is perhaps better thought of as type $D_{2}$, since the visible parabolic in this orbit corresponds to the two nodes of the diagram that form the fork. So if $V_{J}^{\perp}$ is the $D_{n-2}$ summand of $a^{\perp}$ then $J=\{a, b\}$ consists of the two nodes of valency 1 that are adjacent to $c$, the node of valency 3 . A similar statement applies for $D_{4}$ in the case that $V_{J}^{\perp}$ is of type $A_{1}+A_{1}$. In both cases the element $w=r_{c} r_{a} r_{b} r_{c} \in W$ satisfies $w a=b$ and $w b=a$, and since $\Phi_{J}=\{ \pm a, \pm b\}$ we see that $Q=\left\{1, r_{a}, w, w r_{a}\right\} \operatorname{Stab}(a)$. $\operatorname{But} \operatorname{Stab}(a)=\left\langle r_{b}\right\rangle \times W^{\prime}$ and $\operatorname{Stab}(b)=\left\langle r_{a}\right\rangle \times W^{\prime}$, where $W^{\prime}$ is the parabolic subgroup corresponding to the subspace $V_{J}^{\perp}$, and it follows readily that $Q$ stabilizes $\Phi_{J}=\{ \pm a, \pm b\}$, as required.

Continuing the discussion of $D_{n}$, where $n \geqslant 4$, suppose now that $V_{J}^{\perp}$ is an $A_{1}$ component of $a^{\perp}$. Then $V_{J}=\left(V_{J}^{\perp}\right)^{\perp}$ is of type $A_{1}+D_{n-2}$. But the maximal length element of a visible $A_{n-1}$ subsystem containing $a$ takes $a$ to an element of $\Phi_{J}$ but does not normalize $W_{J}$. So our requirement that $Q \subseteq N_{W}\left(W_{J}\right)$ is not met.

Finally, suppose that $\Pi$ is of type $H_{3}$, so that $\operatorname{Stab}(a)$ is of type $A_{1}+A_{1}$. Then $V_{J}^{\perp}$ is of type $A_{1}$, and hence $J$ is of type $A_{1}+A_{1}$. Let $J=\{a, c\}$, and note that $c=w a$ for some $w \in W$. Since $N_{W}\left(W_{J}\right)$ is generated by $W_{J}$ and the central involution of $W$, we see that $c$ is not in the $N_{W}\left(W_{J}\right)$-orbit of $a$. Hence the element $w$ above is in $Q$ but not in $N_{W}\left(W_{J}\right)$, and so our requirements are not met.

We have thus established the following result.

Proposition 26. Let $\Pi$ be the set of simple roots for the finite irreducible Coxeter group $W$, and suppose that $a \in J \subseteq \Pi$. Then $\left\{w \in W \mid w a \in \Phi_{J}\right\}$ is a subset of $N_{W}\left(W_{J}\right)$ if and only if one of the following situations occurs:

(1) $J=\{a\}$;

(2) $J=\Pi$;

(3) $\Pi=\{a, b, c\}$ is of type $C_{3}$, with $m_{a c}=3$ and $m_{c b}=4$, and $J=\{a, b\}$ of type $A_{1}+A_{1}$

(4) $\Pi$ is of type $C_{n}$ and $J=\{a, b\}$ is of type $C_{2}$, with $b$ an end node of $\Pi$;

(5) $\Pi$ is of type $D_{n}$ or $A_{3}$, and $J=\{a, b\}$, where $a$ and $b$ are end nodes that are both adjacent to some $c \in \Pi$.

We return now to investigation of an arbitrary finite rank Coxeter group $W$. The next proposition is an immediate consequence of Proposition 26 and the discussion preceding it.

Proposition 27. Let $a \in J \subseteq L \subseteq \Pi$, and suppose that the group $W_{L}$ is finite and that $W_{J}=\mathrm{FC}\left(r_{a}\right)$. Let $J_{0}$ be the component of $J$ containing $a$ and $L_{0}$ the component 
of $L$ containing $J_{0}$. Then every component of $J$ that is not contained in $L_{0}$ is a component of $L$. Furthermore, if $\{a\} \neq J \cap L_{0} \neq L_{0}$ then $J \cap L_{0}=\{a, b\}$ for some $b$, and one of the following alternatives occurs:

(1) $L_{0}=\{a, c, b\}$ is of type $C_{3}$, with $m_{a c}=3$ and $m_{c b}=4$;

(2) $L_{0}$ is of type $C_{n}$ for some $n \geqslant 3$, with $b$ an end node and $J_{0}=\{a, b\}$ of type $C_{2}$;

(3) $L_{0}$ is of type $A_{3}$ or type $D_{n}$ for some $n \geqslant 4$, the nodes a and b having valency 1 and sharing a common neighbour.

One of the ingredients of alternative (2) of Proposition 27 is that the component of $\mathrm{FC}\left(r_{a}\right)$ containing $a$ is of type $C_{2}$. We shall see that when this situation arises, $\operatorname{Odd}(a)$ has a focus in $\Pi$.

Proposition 28. Suppose that $a \in J \subseteq \Pi$ with $W_{J}=\mathrm{FC}\left(r_{a}\right)$, and let $J_{0}$ be the component of $J$ containing $a$. Suppose that $J_{0}=\{a, b\}$ is of type $C_{2}$. Then either $\operatorname{Odd}(a) \cup\{b\}$ is a spherical component of $\operatorname{EOdd}(a)$, or else $(a, b)$ is a focus of $\operatorname{Odd}(a)$ in $\Pi$.

Proof. We use induction on $k$ to prove that for all $k \geqslant 2$, if $b=c_{1}, a=c_{2}, c_{3}, \ldots, c_{k}$ are simple roots satisfying

(1) $2<m_{c_{i} c_{i+1}}<\infty$ for all $i \in\{1,2, \ldots, k-1\}$, and

(2) $c_{1}, c_{2}, \ldots, c_{k}$ are distinct from each other,

then $\left\{c_{1}, c_{2}, \ldots, c_{k}\right\}$ forms a system of type $C_{k}$. The case $k=2$ is immediately true.

Suppose that $k>2$. The inductive hypothesis tells us that $\left\{c_{1}, c_{2}, \ldots, c_{k-1}\right\}$ is of type $C_{k-1}$. The element $w=v\left[c_{k-1},\left\{c_{k-2}\right\}\right] \ldots v\left[c_{4},\left\{c_{3}\right\}\right] v\left[c_{3},\left\{c_{2}\right\}\right]$ has the property that $w a=w c_{2}=c_{k-1}$, and so if we write $d=w b$ then

$$
r_{d}=w r_{b} w^{-1} \in w \mathrm{FC}\left(r_{a}\right) w^{-1}=\mathrm{FC}\left(c_{k-1}\right),
$$

since it is given that $b \in \mathrm{FC}\left(r_{a}\right)$. But $W_{\left\{c_{k-1}, c_{k}\right\}}$ is finite, and so it follows that $\left\{r_{d}, r_{c_{k-1}}, r_{c_{k}}\right\}$ generates a finite group. Now $d \cdot c_{k-1}=b \cdot a=-\cos (\pi / 4)$ and $c_{k-1} \cdot c_{k}=-\cos (\pi / m)$ for some $m>2$. If $m \geqslant 4$ then

$$
c_{k} \cdot d=c_{k} \cdot\left(b+\sqrt{2} \sum_{i=2}^{k-1} c_{i}\right) \leqslant-\sqrt{2}\left(c_{k} \cdot c_{k-1}\right) \leqslant-1,
$$

whence the reflection subgroup $W_{\left\{r_{d}, r_{c_{k}}\right\}}$ is infinite (by Theorem 10), a contradiction. So $m=3$. If $m_{c_{i} c_{k}}>2$ for any $i \in\{1,2, \ldots, k-2\}$ then

$$
c_{k} \cdot d \leqslant \sqrt{2}\left(c_{k} \cdot c_{k-1}\right)+c_{k} \cdot c_{i}<-1,
$$


again giving a contradiction. So $m_{c_{k} c_{i}}=2$ for all $i \in\{1,2, \ldots, k-2\}$ and $m_{c_{k} c_{k-1}}=$ 3 , and since $\left\{c_{1}, c_{2}, \ldots, c_{k-1}\right\}$ is a system of type $C_{k-1}$ it follows that $\left\{c_{1}, c_{2}, \ldots, c_{k}\right\}$ is a system of type $C_{k}$, as claimed.

If there were $c, d \in \operatorname{Odd}(a)$ with $3<m_{c d}<\infty$ then $b$ together with a minimal length odd-labelled path from $a$ to $\{c, d\}$ would yield $c_{1}, c_{2}, \ldots, c_{k} \in \Pi$ satisfying (1) and (2) above and not forming a system of type $C_{k}$, contradicting the result proved above. The same argument yields a contradiction if $c \in \operatorname{Odd}(a)$ and $d \in \Pi \backslash \operatorname{Odd}(a)$ with $3<m_{c d}<\infty$, unless $\{c, d\}=\{a, b\}$. So all edge labels in $\operatorname{Odd}(a)$ are 3 , if $c, d \in \operatorname{Odd}(a)$ are not adjacent in $\operatorname{Odd}(a)$ then $m_{c d} \in\{2, \infty\}$, and if $c \in \operatorname{Odd}(a)$ and $d \in \Pi \backslash \operatorname{Odd}(a)$ then $m_{c d} \in\{2, \infty\}$ unless $\{c, d\}=\{a, b\}$. Furthermore, any circuit in $\operatorname{Odd}(a)$ would similarly yield a contradiction (by combining the circuit with a minimal finite-labelled path connecting it to $b)$. So $\operatorname{Odd}(a)$ is tree.

For each $c \in \operatorname{Odd}(a)$ let $C[b . . c] \subseteq \Pi$ consist of $b$ and the unique path from $a$ to $c$ in $\operatorname{Odd}(a)$. The discussion above shows that $C[b . . c]$ is always of type $C$. Now suppose that $c \in \operatorname{Odd}(a)$ and $e \in \Pi \backslash C[b . . c]$ with $m_{c e}=2$. Write $C[b . . c]=\left\{c_{1}, c_{2}, \ldots, c_{k}\right\}$, with $c_{1}=b$ and $c_{k}=c$, and let $d=b+\sqrt{2} \sum_{i=2}^{k} c_{i}$. An argument similar to one used above shows that $r_{d} \in \mathrm{FC}(c)$, and hence $W_{\{d, c, e\}}$ is finite. So $d \cdot e>-1$. If $c_{i} \cdot e \neq 0$ then $c_{i} \cdot e \leqslant-1 / 2$; so it follows that there is at most one $i$ with $c_{i} \cdot e \neq 0$. Suppose, for a contradiction, that there is exactly one such $i$. If $i>1$ then $d \cdot e=\sqrt{2}\left(c_{i} \cdot e\right)$, and so $c_{i} \cdot e>-1 / \sqrt{2}$. Hence $m_{c_{i} e}=3$, and $d \cdot e=-1 / \sqrt{2}$. But this means that the edges $\{c, d\}$ and $\{d, e\}$ of the Coxeter graph of $\{d, c, e\}$ are both labelled 4, contradicting the fact that $W_{\{d, c, e\}}$ is finite. So we must have $i=1$, and finiteness of $W_{\{c, d, e\}}$ forces $b \cdot e=d \cdot e=-1 / 2$. But now if we put $L=\{e\} \cup J$ then, in the notation of Proposition 27, we have that $L_{0}=\{e, b, a\}$ is of type $C_{3}$ with $J \cap L_{0}=\{b, a\}$ of type $C_{2}$, and Proposition 27 shows that this is not possible. We conclude that if $e \in \Pi$ has the property that $m_{c e}=2$ for some $c \in \operatorname{Odd}(a)$ then $m_{d e}=2$ for all $d \in C[b . . c]$. In particular, if $e \in \Pi \backslash(\operatorname{Odd}(a) \cup\{b\})$ and $m_{c e} \neq \infty$ for some $c \in \operatorname{Odd}(a)$ then $m_{c e}=2$, as shown above, and so $m_{d e}=2$ for all $d \in C[b . . c]$.

All that remains to prove now is that if $c, d \in \operatorname{Odd}(a)$ with $c \notin C[b . . d]$ and $d \notin C[b . . c]$, then $m_{c d}=\infty$. Since $c$ and $d$ are not adjacent in $\operatorname{Odd}(a)$ the only alternative is that $m_{c d}=2$; so suppose, for a contradiction, that this holds. Choose the vertex $e \in \operatorname{Odd}(a)$ on the (unique) path from $c$ to $d$ such that the distance from $e$ to $a$ is minimal. Let $c^{\prime}, d^{\prime}$ be the neighbours of $e$ in the path from $c$ to $d$, with $c^{\prime}$ between $e$ and $c$ and $d^{\prime}$ between $e$ and $d$. Then $c^{\prime} \in C[b . . c]$, and since $m_{c d}=2$ it follows that $m_{c^{\prime} d}=2$. Now since $d^{\prime} \in C[b . . d]$ and $m_{c^{\prime} d}=2$ it follows that $m_{c^{\prime} d^{\prime}}=2$. Thus the set $L \subseteq \Pi$ consisting of $c^{\prime}$ and $d^{\prime}$ and the vertices on the path from $a$ to $e$ form a system of type $D$ (or $A_{3}$ if $e=a$ ). So $L$ is spherical, and since $b \in \mathrm{FC}\left(r_{a}\right)$ it follows that $L \cup\{b\}$ is spherical also. But this is impossible since $L \cup\{b\}$ is connected, has an edge labelled 4 (namely, $\{b, a\}$ ), and has a vertex of valency 3 (namely $e$ ). 
The situation of alternative (3) of Proposition 27 is very similar to that of alternative (2), and in this case it turns out that $\operatorname{Odd}(a)$ has a half-focus in $\Pi$.

Proposition 29. Suppose that $a \in J \subseteq \Pi$ with $W_{J}=\mathrm{FC}\left(r_{a}\right)$ and $\{a\}$ a component of $J$, and suppose that $J \cap \operatorname{Odd}(a) \neq\{a\}$. Then either $\operatorname{Odd}(a)$ is a spherical component of $\operatorname{EOdd}(a)$, or else there exists an element $b \in \operatorname{Odd}(a)$ such that $\{a, b\}$ is a half focus of $\operatorname{Odd}(a)$ in $\Pi$.

Proof. Let $b \in(J \cap \operatorname{Odd}(a)) \backslash\{a\}$, and let $w \in W$ with $w a=b$. Then $w \in N_{W}\left(W_{J}\right)$, by Proposition 25, and so

$$
\mathrm{FC}\left(r_{b}\right)=\mathrm{FC}\left(w r_{a} w^{-1}\right)=w \mathrm{FC}\left(r_{a}\right) w^{-1}=w W_{J} w^{-1}=W_{J} .
$$

Moreover, $w \Phi_{J}=\Phi_{J}$, and since $a \cdot c=0$ for all $c \in \Phi_{J} \backslash\{a\}$, it follows that $w a \cdot d=0$ for all $d \in \Phi_{J} \backslash\{w a\}$. So $\{b\}$ is a component of $J$. Note that $m_{a b}=2$, since $a$ and $b$ are in different components of $J$.

Let $c \in \Pi \backslash\{a, b\}$, and suppose first of all that $2<m_{b c}<\infty$. Since $\{b, c\}$ is spherical and $W_{J}=\mathrm{FC}\left(r_{b}\right)$ it follows that $J \cup\{c\}$ is spherical. Let $L=J \cup\{c\}$ and let $L_{0}$ be the component of $L$ containing $a$. By Proposition 27, every component of $J$ that is not contained in $L_{0}$ is a component of $L$. But $b$ is adjacent to $c$ in $L$; so $\{b\}$ is not a component of $L$, and it follows that $b \in L_{0}$. Now $\{a\} \neq J \cap L_{0}$, since $b \in J \cap L_{0}$, and $J \cap L_{0} \neq L_{0}$, since $c \in L_{0}$ and $c \notin J$ (since $\{b\}$ is a component of $J$ ). Furthermore, the conditions of alternative (2) of Proposition 27 are not satisfied, since $a$ and $b$ are not adjacent in $J$. So either alternative (1) or alternative (3) must hold, and since $c$ is the only element of $L$ not in $J$ it follows that $L_{0}=\{a, c, b\}$, with $m_{a c}=3$. But a symmetrical argument, with the roles of $a$ and $b$ reversed, shows that every $d \in \Pi$ with $2<m_{a d}<\infty$ has the property that $m_{b d}=3$. So $m_{a c}=m_{b c}=3$, and $\{a, c, b\}$ is of type $A_{3}$.

Now suppose that $m_{b c}=2$. Again since $\{b, c\}$ is spherical it follows that $J \cup\{c\}$ is spherical, and so $m_{a c}<\infty$. If $m_{a c}>2$ then, as we have just observed, it follows that $m_{b c}=3$, contrary to our assumption that $m_{b c}=2$. So $m_{a c}=m_{b c}=2$, and we have now shown that whenever $m_{b c}<\infty$ we have $m_{a c}=m_{b c} \in\{2,3\}$. Since a symmetrical argument gives the same conclusion whenever $m_{a c}<\infty$, we conclude also that $m_{a c}=\infty$ if and only if $m_{b c}=\infty$.

We now use induction on $k$ to prove that for all $k \geqslant 3$, if $b=c_{1}, a=$ $c_{2}, c_{3}, \ldots, c_{k}$ are simple roots satisfying

(1) $2<m_{c_{i} c_{i+1}}<\infty$ for all $i \in\{2,3, \ldots, k-1\}$, and

(2) $c_{1}, c_{2}, \ldots, c_{k}$ are distinct from each other,

then $\left\{c_{1}, c_{2}, \ldots, c_{k}\right\}$ forms a system of type $D_{k}$ or $A_{3}$. The case $k=3$ follows from what we have proved above. 
Suppose that $k>3$. The inductive hypothesis tells us that $\left\{c_{1}, c_{2}, \ldots, c_{k-1}\right\}$ is of type $D_{k-1}$ (or $A_{3}$ if $k=4$ ). The element $w=v\left[c_{k-1},\left\{c_{k-2}\right\}\right] \ldots v\left[c_{4},\left\{c_{3}\right\}\right] v\left[c_{3},\left\{c_{2}\right\}\right]$ has the property that $w a=w c_{2}=c_{k-1}$, and so if we write $d=w b$ then

$$
r_{d}=w r_{c_{1}} w^{-1} \in w \operatorname{FC}\left(r_{a}\right) w^{-1}=\operatorname{FC}\left(c_{k-1}\right),
$$

since it is given that $b \in \mathrm{FC}\left(r_{a}\right)$. But $W_{\left\{c_{k-1}, c_{k}\right\}}$ is finite, and so it follows that $\left\{r_{d}, r_{c_{k-1}}, r_{c_{k}}\right\}$ generates a finite group. Now $d \cdot c_{k-1}=b \cdot a=0$ and $c_{k-1} \cdot c_{k}=$ $-\cos (\pi / m)$ for some $m>2$. If $c_{k} \cdot c_{i} \neq 0$ for some $i \in\{1,2, \ldots, k-2\}$ then

$$
c_{k} \cdot d=c_{k} \cdot\left(c_{1}+c_{2}+c_{k-1}+2 \sum_{i=3}^{k-2} c_{i}\right) \leqslant-\left(\frac{1}{2}+\cos \frac{\pi}{m}\right) \leqslant-1,
$$

whence the reflection subgroup $W_{\left\{r_{d}, r_{c_{k}}\right\}}$ is infinite (by Theorem 10), a contradiction. So $c_{k} \cdot d=c_{k-1} \cdot c_{k}=-\cos \frac{\pi}{m}$. Since the reflection subgroup generated by $\left\{r_{d}, r_{c_{k-1}}, r_{c_{k}}\right\}$ is finite it follows that $m=3$. So we have shown that $m_{c_{k} c_{k-1}}=3$ and $m_{c_{k} c_{i}}=2$ for $i<k-1$, and since $\left\{c_{1}, c_{2}, \ldots, c_{k-1}\right\}$ is a system of type $D_{k-1}$ it follows that $\left\{c_{1}, c_{2}, \ldots, c_{k}\right\}$ is a system of type $D_{k}$, as claimed.

Note that $\operatorname{Odd}(a) \backslash\{b\}$ and $\operatorname{Odd}(b) \backslash\{a\}$ are both connected, since each element $c \in \operatorname{Odd}(a)$ that is adjacent to $a$ is also adjacent to $b$, and vice versa. If there were $c, d \in \operatorname{Odd}(a) \backslash\{b\}$ with $3<m_{c d}<\infty$ then $b$ together with a minimal length odd-labelled path from $a$ to $\{c, d\}$ would yield $c_{1}, c_{2}, \ldots, c_{k} \in \Pi$ satisfying (1) and (2) above and not forming a system of type $D_{k}$, contradicting the result proved above. The same argument yields a contradiction whenever $c \in \operatorname{Odd}(a) \backslash\{a, b\}$ and $d \in \Pi \backslash \operatorname{Odd}(a)$ with $3<m_{c d}<\infty$. So all edge labels in $\operatorname{Odd}(a)$ are 3 , if $c, d \in \operatorname{Odd}(a)$ are not adjacent in $\operatorname{Odd}(a)$ then $m_{c d} \in\{2, \infty\}$, and if $c \in \operatorname{Odd}(a)$ and $d \in \Pi \backslash \operatorname{Odd}(a)$ then $m_{c d} \in\{2, \infty\}$. Furthermore, any circuit in $\operatorname{Odd}(a) \backslash\{b\}$ would similarly yield a contradiction (by combining the circuit with a minimal finitelabelled path connecting it to $b$ ). $\operatorname{So} \operatorname{Odd}(a) \backslash\{b\}$ is tree. Of course, $\operatorname{Odd}(b) \backslash\{a\}$ is also a tree, by the same argument.

For each $c \in \operatorname{Odd}(a) \backslash\{a, b\}$ let $D[a, b . . c] \subseteq \Pi$ consist of $b$ and the unique path from $a$ to $c$ in $\operatorname{Odd}(a) \backslash\{b\}$. The discussion above shows that $D[a, b . . c]$ is of type $D$. Now suppose that $c \in \operatorname{Odd}(a) \backslash\{a, b\}$ and $e \in \Pi \backslash D[a, b . . c]$ with $m_{c e}=2$. Write $D[a, b . . c]=\left\{c_{1}, c_{2}, \ldots, c_{k}\right\}$, with $c_{1}=b, c_{2}=a$ and $c_{k}=c$, and let $d=c_{1}+c_{2}+c_{k}+2 \sum_{i=3}^{k-1} c_{i}$. An argument similar to one used above shows that $r_{d} \in \mathrm{FC}(c)$, and hence $W_{\{d, c, e\}}$ is finite. So $d \cdot e>-1$. If $c_{i} \cdot e \neq 0$ then $c_{i} \cdot e \leqslant-1 / 2$; so it follows that $\left\{i \mid c_{i} \cdot e \neq 0\right\}$ is a subset of $\{1,2, k\}$ with at most one element. But $c_{k} \cdot e=0$ since $m_{c e}=2$, and $c_{1} \cdot e=c_{2} \cdot e$ since $m_{a f}=m_{b f}$ for all $f \in \Pi$. So $c_{i} \cdot e=0$ for all $i \in\{1,2, \ldots, k\}$. In particular, if $e \in \Pi \backslash \operatorname{Odd}(a)$ and $m_{c e} \neq \infty$ for some $c \in \operatorname{Odd}(a)$ then $m_{c e}=2$, as shown above, and so $m_{d e}=2$ for all $d \in D[a, b . . c]$. 
All that remains to prove now is that if $c, d \in \operatorname{Odd}(a) \backslash\{a, b\}$ with $c \notin D[a, b . . d]$ and $d \notin D[a, b . . c]$, then $m_{c d}=\infty$. Since $c$ and $d$ are not adjacent in $\operatorname{Odd}(a)$ the only alternative is that $m_{c d}=2$; so suppose, for a contradiction, that this holds. Choose the vertex $e \in \operatorname{Odd}(a) \backslash\{b\}$ on the (unique) path from $c$ to $d$ such that the distance from $e$ to $a$ is minimal. Let $c^{\prime}, d^{\prime}$ be the neighbours of $e$ in the path from $c$ to $d$, with $c^{\prime}$ between $e$ and $c$ and $d^{\prime}$ between $e$ and $d$. Then $c^{\prime} \in C[b . . c]$, and since $m_{c d}=2$ it follows that $m_{c^{\prime} d}=2$. Now since $d^{\prime} \in C[b . . d]$ and $m_{c^{\prime} d}=2$ it follows that $m_{c^{\prime} d^{\prime}}=2$. Thus the set $L \subseteq \Pi$ consisting of $c^{\prime}$ and $d^{\prime}$ and the vertices on the path from $a$ to $e$ form a system of type $D_{k}$, or $A_{3}$ if $e=a$. So $L$ is spherical, and since $b \in \mathrm{FC}\left(r_{a}\right)$ it follows that $L \cup\{b\}$ is spherical also. If $L=A_{3}$ then $m_{a c^{\prime}}=m_{a d^{\prime}}=3$, and since $m_{b c^{\prime}}=m_{a c^{\prime}}$ and $m_{b d^{\prime}}=m_{a d^{\prime}}$ we see that $L \cup\{b\}$ is of type $\widetilde{A}_{3}$, contradicting the fact that $L \cup\{b\}$ is spherical. Similarly if $L$ is of type $D_{k}$ then $L \cup\{b\}$ is of type $\widetilde{D}_{k}$, again giving a contradiction.

We also need to obtain further information about the situation of alternative (1) of Proposition 27. So for the next three lemmas we assume that $a \in J \subseteq L \subseteq \Pi$ with $L$ spherical and $W_{J}=\mathrm{FC}\left(r_{a}\right)$, and there exist $b \in J$ and $c \in L \backslash J$ such that $L_{0}=\{a, c, b\}$ is a component of $L$ of type $C_{3}$, with $m_{a c}=3$ and $m_{c b}=4$.

Lemma 30. For all $e \in \Pi \backslash\{a, c, b\}$, either $m_{c e}=m_{a e}=m_{b e}=2$ or $m_{c e}=\infty$. Moreover, $J \cap \operatorname{Odd}(a)=\{a\}$.

Proof. If $J \cap \operatorname{Odd}(a) \neq\{a\}$ then, since $\{a\}$ is a component of $J$, Proposition 29 applies, and it follows in particular that no vertex in $\operatorname{Odd}(a)$ lies on an edge with finite label different from 3. This contradicts $m_{b c}=4$. So $J \cap \operatorname{Odd}(a)=\{a\}$.

Suppose that $e \in \Pi \backslash\{a, c, b\}$ with $m_{c e}<\infty$. The group $r_{c} r_{a} W_{\{c, e\}} r_{a} r_{c}$ is finite and contains $r_{c} r_{a} W_{\{c\}} r_{a} r_{c}=W_{\{a\}}$; so there exists a maximal finite subgroup $G$ of $W$ containing $r_{a}$ and the reflection along $\left(r_{c} r_{a}\right) e$. Since $r_{b} \in \mathrm{FC}\left(r_{a}\right) \subseteq G$ it follows that $W_{\left\{b,\left(r_{c} r_{a}\right) e\right\}}$ is finite, and hence so is $W_{\left\{\left(r_{a} r_{c}\right) b, e\right\}}=r_{a} r_{c} W_{\left\{b,\left(r_{c} r_{a}\right) e\right\}} r_{c} r_{a}$. Hence

$$
(b+\sqrt{2} c+\sqrt{2} a) \cdot e=\left(r_{a} r_{c}\right) b \cdot e>-1 .
$$

Assume, for a contradiction, that $m_{c e} \neq 2$. Then $c \cdot e \leqslant-1 / 2<-1 / 2 \sqrt{2}$, and so $(b+\sqrt{2} a) \cdot e>-1 / 2$, giving a contradiction if either $m_{b e} \neq 2$ or $m_{a e} \neq 2$. So $b \cdot e=a \cdot e=0$, and the inequality 4.1 above gives $c \cdot e>-1 / \sqrt{2}$. So $m_{c e}=3$. But now $W_{\{a, c, e\}}$ is of type $A_{3}$, hence finite, and hence contained in a maximal finite subgroup that also contains $\mathrm{FC}\left(r_{a}\right)=W_{J}$. Since $b \in J$ it follows that $\{a, c, e, b\}$ is spherical, which is false since it is of type $\widetilde{B}_{3}$. So $m_{c e}=2$, and it remains to show that $m_{a e}=m_{b e}=2$.

Since $c \cdot e=0$ we deduce from 4.1 that $(b+\sqrt{2} a) \cdot e>-1$, and in particular it follows that $m_{a e}$ is 2 or 3 . In either case $\{e, a, c\}$ is spherical (of type $A_{3}$ or $A_{1}+A_{2}$ ), and so $\{e, a, c, b\}$ is also spherical (since $r_{b} \in \mathrm{FC}\left(r_{a}\right)$ ). If either $m_{a e} \neq 2$ or $m_{b e} \neq 2$ 
then applying Proposition 27 with $L^{\prime \prime}=J \cup\{e, c\}$ in place of $L$ yields a contradiction, since if $L_{0}^{\prime \prime}$ is the component of $L^{\prime \prime}$ containing $a$ then $\{a, b\} \subseteq L_{0}^{\prime \prime} \cap J \neq L_{0}^{\prime \prime}$ (since $c \notin J)$, but $L_{0}^{\prime \prime}$ is not of type $C_{3}$ or $D_{n}$ since it contains $\{a, c, b, e\}$. So $m_{a e}=m_{b e}=2$, as required.

Lemma 31. Let $J^{\prime}=J \backslash\{a\}$ and let $d \in \operatorname{Odd}(a)$. Then $m_{c d} \neq 2$. If $m_{d b^{\prime}} \neq 2$ for some $b^{\prime} \in J^{\prime}$ then $m_{d b^{\prime}}=4$, and there is a unique $a^{\prime}$ adjacent to $d$ in $\operatorname{Odd}(a)$; moreover, $\left\{a^{\prime}, d, b^{\prime}\right\}$ is of type $C_{3}$, and $\mathrm{FC}\left(r_{a^{\prime}}\right)=W_{J^{\prime} \cup\left\{a^{\prime}\right\}}$. On the other hand, if $m_{d b^{\prime}}=2$ for all $b^{\prime} \in J^{\prime}$ then $\mathrm{FC}\left(r_{d}\right)=W_{J^{\prime} \cup\{d\}}$.

Proof. We use induction on the distance from $d$ to $a$ in $\operatorname{Odd}(a)$. Observe that if $d=a$ then $m_{d b^{\prime}}=2$ for all $b^{\prime} \in J^{\prime}$, since $\{a\}$ is a component of $J$, and we have $\mathrm{FC}\left(r_{d}\right)=W_{J}=W_{J^{\prime} \cup\{d\}}$ and $m_{c d}=3 \neq 2$, as required.

Suppose now that $d \neq a$, and let $a=d_{1}, d_{2}, \ldots, d_{k}=d$ be a minimal length path from $a$ to $d$ in $\operatorname{Odd}(a)$. If $2 \leqslant i \leqslant k-1$ then $d_{i}$ does not have valency 1 in $\operatorname{Odd}(a)$, and so $m_{d_{i} b^{\prime}}=2$ for all $b^{\prime} \in J^{\prime}$, by the inductive hypothesis. The same is true for $i=1$, since $\{a\}$ is a component of $J$.

We prove first that $m_{c d} \neq 2$. Assuming, for a contradiction, that $m_{c d}=2$, then clearly $d \notin\{a, c, b\}$, and Lemma 30 tells us that $m_{a d}=2$ and $m_{b d}=2$. It follows that $m_{b f}=2$ for all $f$ in the set $M=\left\{d_{1}, d_{2}, \ldots, d_{k}\right\}$, since $\left\{d_{i}\right\}$ is a component of $J^{\prime} \cup\left\{d_{i}\right\}$ when $1 \leqslant i \leqslant k-1$. So $w b=b$ for all $w \in W_{M}$. Furthermore, since $d$ and $a$ lie in the same connected component of $\Omega(\Pi)$, we can choose $w \in W_{M}$ such that $w d=a$. Now since $w c \cdot a=c \cdot d=0$ we see that the reflection $r_{w c}$ centralizes $r_{a}$, and hence normalizes $\mathrm{FC}\left(r_{a}\right)=W_{J}$. By Lemma 11 it follows that either $w c \in \Phi_{J}$ or $w c \cdot e=0$ for all $e \in J$. But $w c \cdot b=c \cdot w^{-1} b=c \cdot b \neq 0$; so we must have $w c \in \Phi_{J}$, and hence $c \in w^{-1} \Phi_{J} \subseteq \Phi_{J \cup M}$. So $c \in M$, contradicting $m_{b f}=2$ for all $f \in M$. So $m_{c d} \neq 2$.

Write $a^{\prime}=d_{k-1}$ and $\widetilde{J}=J^{\prime} \cup\left\{a^{\prime}\right\}$. Note that $\left\{a^{\prime}\right\}$ is a component of $\widetilde{J}$, and $\mathrm{FC}\left(r_{a^{\prime}}\right)=W_{\widetilde{J}}$ (by the inductive hypothesis). Now since $\left\{d, a^{\prime}\right\}$ is spherical, $\widetilde{L}=$ $\widetilde{J} \cup\{d\}$ is spherical also. Let $\widetilde{L}_{0}$ be the component of $\widetilde{L}$ containing $a^{\prime}$.

Consider first the case that $m_{d b^{\prime}}=2$ for all $b^{\prime} \in J^{\prime}$. Since also $m_{a^{\prime} b^{\prime}}=2$ for all $b^{\prime} \in J^{\prime}$, it follows that $r_{d}$ and $r_{a^{\prime}}$ both fix all elements of $J^{\prime}$. Since $v=v\left[d,\left\{a^{\prime}\right\}\right] \in$ $W_{\left\{a^{\prime}, d\right\}}$ satisfies $v a^{\prime}=d$, we conclude that

$$
\mathrm{FC}\left(r_{d}\right)=v \mathrm{FC}\left(r_{a^{\prime}}\right) v^{-1}=v W_{J^{\prime} \cup\left\{a^{\prime}\right\}} v^{-1}=W_{v J^{\prime} \cup\left\{v a^{\prime}\right\}}=W_{J^{\prime} \cup\{d\}},
$$

as required.

Now suppose that $m_{d b^{\prime}} \neq 2$ for some $b^{\prime} \in J^{\prime}$. Then $b^{\prime} \in \widetilde{J} \cap \widetilde{L}_{0}$, and so $\left\{a^{\prime}\right\} \neq$ $\widetilde{J} \cap \widetilde{L}_{0} \neq \widetilde{L}_{0}$. Applying Proposition 27, we see that the situation of alternative (1) must hold: alternative (2) is ruled out since $a^{\prime}$ is a component of $\widetilde{J}$, and alternative (3) is ruled out since $J^{\prime} \cap \operatorname{Odd}(a)=\emptyset$. Hence $\widetilde{L}_{0}=\left\{a^{\prime}, d, b^{\prime}\right\}$ is of type $C_{3}$, with $m_{d b^{\prime}}=4$ and $m_{d a^{\prime}}=3$. Furthermore, Lemma 30 tells us that $m_{d e} \in\{2, \infty\}$ for all $e \in \Pi \backslash\left\{a^{\prime}, d, b^{\prime}\right\}$; so $a^{\prime}$ is the unique neighbour of $d$ in $\operatorname{Odd}(a)$, as required. 
Lemma 32. Let $e \in \operatorname{EOdd}(a) \backslash \operatorname{Odd}(a)$ with $e \neq b$. Then $m_{b e}=2$.

Proof. If $e \in J$ then it is clear that $m_{b e}=2$, since $b$ is a component of $J$. So we may assume that $e \notin J$.

As above, write $J^{\prime}=J \backslash\{a\}$. Since $e \in \operatorname{EOdd}(a)$ there exists a $d \in \operatorname{Odd}(a)$ with $m_{d e}$ even. If $d$ is adjacent to some $b^{\prime} \in J^{\prime}$ then, by Lemma 31, there is a unique $a^{\prime} \in \operatorname{Odd}(a)$ adjacent to $d$; furthermore, $\left\{a^{\prime}, d, b^{\prime}\right\}$ is of type $C_{3}$, and $\mathrm{FC}\left(r_{a^{\prime}}\right)=W_{J^{\prime} \cup\left\{a^{\prime}\right\}}$. By Lemma 30, since $m_{d e} \neq \infty$ it follows that $m_{a^{\prime} e}=m_{d e}=2$. On the other hand, if $d$ is not adjacent to any element of $J^{\prime}$ then Lemma 31 tells us that $\mathrm{FC}\left(r_{d}\right)=W_{J^{\prime} \cup\{d\}}$. So in either case there is an $a^{\prime} \in \operatorname{Odd}(a)$ with $m_{a^{\prime} e}$ even and $\mathrm{FC}\left(r_{a^{\prime}}\right)=W_{J^{\prime} \cup\left\{a^{\prime}\right\}}$.

Choose such an $a^{\prime}$. Since $m_{a^{\prime} e}$ is even, $v\left[e,\left\{a^{\prime}\right\}\right] a^{\prime}=a^{\prime}$; moreover $v\left[e,\left\{a^{\prime}\right\}\right]$ is the reflection along some root $f=\lambda e+\mu a^{\prime}$. Note that $f \cdot a^{\prime}=0$, and hence $\lambda \neq 0$. Since $e \notin J$ it follows that $f \notin \Phi_{J}$. But $r_{f}$ centralizes $r_{a^{\prime}}$, and hence normalizes $\mathrm{FC}\left(r_{a^{\prime}}\right)=W_{J^{\prime} \cup\left\{a^{\prime}\right\}}$. By Lemma 11 it follows that $f \cdot b=0$. But also $a^{\prime} \cdot b=0$, since $\left\{a^{\prime}\right\}$ and $\{b\}$ are distinct components of $J^{\prime} \cup\left\{a^{\prime}\right\}$; so it follows that $e \cdot b=0$. Thus $m_{b e}=2$, as required.

Lemmas 30, 31 and 32 combine to yield the following result.

Proposition 33. Suppose that $a \in J \subseteq L \subseteq \Pi$, with $L$ spherical and $\mathrm{FC}\left(r_{a}\right)=W_{J}$, and let $L_{0}$ be the component of $L$ containing $a$. Suppose that $L_{0}=\{a, c, b\}$ is of type $C_{3}$, with $m_{a c}=3$ and $m_{b c}=4$, and $J \cap L_{0}=\{a, b\}$. Then $b$ is a $C_{3}$-neighbour of $\operatorname{Odd}(a)$. Furthermore, $J \cap \operatorname{Odd}(a)=\{a\}$, and if $a^{\prime} \in \operatorname{Odd}(a)$ is not adjacent to any $C_{3}$-neighbour of $\operatorname{Odd}(a)$ then $\mathrm{FC}\left(r_{a^{\prime}}\right)=W_{J^{\prime} \cup\left\{a^{\prime}\right\}}$, where $J^{\prime}=J \backslash\{a\}$.

Proof. If $m_{b d} \neq 2$ for some $d \in \operatorname{Odd}(a)$, then $m_{d b}=4$, by Lemma 31. There is at least one $d \in \operatorname{Odd}(a)$ such that $m_{b d}=4$, namely $d=c$. Lemma 31 tells us that for each $d \in \operatorname{Odd}(a)$ with $m_{b d}=4$ there is an $a^{\prime} \in \operatorname{Odd}(a)$ such that $\left\{a^{\prime}, d, b\right\}$ is a system of type $C_{3}$. Moreover, by Lemma 30, if $e \in \Pi \backslash(\operatorname{Odd}(a) \cup\{b\})$ then either $m_{d e}=\infty$ or $m_{a e}=m_{b e}=m_{d e}=2$, while if $e \in \operatorname{Odd}(a) \backslash\{a, c\}$ then $m_{d e}=\infty$, since $m_{d e} \neq 2$ by Lemma 31. And if $e \in \operatorname{EOdd}(a) \backslash(\operatorname{Odd}(a) \cup\{b\})$ then $m_{b e}=2$, by Lemma 32. So $b$ satisfies all the requirements of a $C_{3}$-neighbour of $M=\operatorname{Odd}(a)$, as specified in Definition 4.

It now follows from Lemma 31 that if $a^{\prime} \in \operatorname{Odd}(a)$ is adjacent to some $b^{\prime} \in J^{\prime}$ then $b^{\prime}$ is a $C_{3}$-neighbour of $\operatorname{Odd}(a)$, and if $a^{\prime}$ is not adjacent to any such $b^{\prime}$ then $\mathrm{FC}\left(r_{a^{\prime}}\right)=W_{J^{\prime} \cup\left\{a^{\prime}\right\}}$. Finally, $J \cap \operatorname{Odd}(a)=\{a\}$, by Lemma 30 .

Let $a, a^{\prime} \in \Pi$, and suppose that $w \in W$ has the property that $w a=a^{\prime}$. By Proposition 12 there exist $a_{i} \in \operatorname{Odd}(a)$ and $c_{i} \in \Pi$ such that

(i) $a_{1}=a$ and $a_{k+1}=a^{\prime}$,

(ii) $m_{c_{i} a_{i}} \neq \infty$ and $v\left[c_{i},\left\{a_{i}\right\}\right] a_{i}=a_{i+1}$, for all $i \in\{1,2, \ldots, k\}$,

(iii) $w=v\left[c_{k},\left\{a_{k}\right\}\right] \ldots v\left[c_{2},\left\{a_{2}\right\}\right] v\left[c_{1},\left\{a_{1}\right\}\right]$. 
Now let $b$ be a $C_{3}$-neighbour of $\operatorname{Odd}(a)$. For each $c \in \operatorname{Odd}(a)$ that is adjacent to $b$, define $X(c)=b+\sqrt{2} c+\sqrt{2} \tilde{a}$, where $\tilde{a}$ is the unique neighbour of $c$ in $\operatorname{Odd}(a)$, and for each $c \in \operatorname{Odd}(a)$ that is not adjacent to $b$, define $X(c)=b$. We show that $v\left[c_{i},\left\{a_{i}\right\}\right] X\left(a_{i}\right)=X\left(a_{i+1}\right)$ for all $i \in\{1,2, \ldots k\}$.

Suppose first that neither $c_{i}$ nor $a_{i}$ is adjacent to $b$. Then $X\left(a_{i}\right)=b$, and since $a_{i+1} \in\left\{a_{i}, c_{i}\right\}$ we have that $X\left(a_{i+1}\right)=b$ also. Since $r_{a_{i}}$ and $r_{c_{i}}$ both fix $b$, and $v\left[c_{i},\left\{a_{i}\right\}\right] \in W_{\left\{a_{i}, c_{i}\right\}}$, it follows that

$$
v\left[c_{i},\left\{a_{i}\right\}\right] X\left(a_{i}\right)=v\left[c_{i},\left\{a_{i}\right\}\right] b=b=X\left(a_{i+1}\right),
$$

as required.

Next, suppose that $c_{i}$ is adjacent to $b$, but $a_{i}$ is not adjacent to $b$. Since $m_{c_{i}} a_{i} \neq \infty$ and $a \in \operatorname{Odd}(a)$ it follows that $c_{i} \in \operatorname{EOdd}(a)$. Since $b$ is a $C_{3}$-neighbour of $\operatorname{Odd}(a)$, it is not adjacent to any element of $\operatorname{EOdd}(a) \backslash \operatorname{Odd}(a)$; so $c_{i} \in \operatorname{Odd}(a)$, and, moreover, $m_{c_{i} e}=\infty$ for all $e \in \operatorname{Odd}(a) \backslash\left\{c_{i}\right\}$ apart from the unique neighbour of $c_{i} \operatorname{in} \operatorname{Odd}(a)$. So $a_{i}$ is this unique neighbour, $m_{c_{i} a_{i}}=3$, and $a_{i+1}=v\left[c_{i},\left\{a_{i}\right\}\right] a_{i}=c_{i}$. Moreover, $m_{c_{i} b}=4$ and $m_{a_{i} b}=2$. So

$$
v\left[c_{i},\left\{a_{i}\right\}\right] X\left(a_{i}\right)=r_{a_{i}} r_{c_{i}} b=b+\sqrt{2} c_{i}+\sqrt{2} a_{i}=X\left(c_{i}\right)=X\left(a_{i+1}\right)
$$

as required.

Now suppose that $a_{i}$ is adjacent to $b$, and let $\tilde{a}$ be the unique neighbour of $a_{i}$ in $\operatorname{Odd}(a)$. Since $m_{a_{i} e}=\infty$ for all $e \in \operatorname{Odd}(a) \backslash\left\{a_{i}, \tilde{a}\right\}$, if $c_{i} \in \operatorname{Odd}(a)$ then $c_{i}=\tilde{a}$. In this case we see that

$$
v\left[c_{i},\left\{a_{i}\right\}\right] X\left(a_{i}\right)=r_{a_{i}} r_{c_{i}}\left(b+\sqrt{2} c_{i}+\sqrt{2} a_{i}\right)=b=X\left(c_{i}\right)=X\left(a_{i+1}\right),
$$

since $a_{i+1}=v\left[c_{i},\left\{a_{i}\right\}\right] a_{i}=c_{i}$. If $c_{i}=b$ then $v\left[c_{i},\left\{a_{i}\right\}\right]=r_{b} r_{a_{i}} r_{b}$, which fixes both $a_{i}$ and $X\left(a_{i}\right)=b+\sqrt{2} a_{i}+\sqrt{2} \tilde{a}$. So $v\left[c_{i},\left\{a_{i}\right\}\right] X\left(a_{i}\right)=X\left(a_{i+1}\right)$ in this case too. Finally, suppose that $c_{i} \notin \operatorname{Odd}(a) \cup\{b\}$. Since $m_{c_{i} a_{i}} \neq \infty$ we must have $m_{c_{i} \tilde{a}}=m_{c_{i} a_{i}}=m_{c_{i} b}=2$, (by the definition of a $C_{3}$-neighbour). So

$$
v\left[c_{i},\left\{a_{i}\right\}\right] X\left(a_{i}\right)=r_{c_{i}}\left(b+\sqrt{2} c_{i}+\sqrt{2} \tilde{a}\right)=b+\sqrt{2} c_{i}+\sqrt{2} \tilde{a}=X\left(a_{i+1}\right)
$$

since $a_{i+1}=r_{c_{i}} a_{i}=a_{i}$.

We have now covered all cases, and shown that $v\left[c_{i},\left\{a_{i}\right\}\right] X\left(a_{i}\right)=X\left(a_{i+1}\right)$ for all $i \in\{1,2, \ldots k\}$. By a trivial induction it follows that $X\left(a_{k+1}\right)=w X\left(a_{1}\right)$.

Thus we have established the following result.

Lemma 34. Let $a \in \Pi$ and $w \in W$ such that $w a \in \Pi$. Suppose that $b$ is $a$ $C_{3}$-neighbour of $\operatorname{Odd}(a)$ that is not adjacent to $a$. Then

$$
w b= \begin{cases}b & \text { if wa is not adjacent to } b, \\ b+\sqrt{2} w a+\sqrt{2} \tilde{a} & \text { if wa is adjacent to } b,\end{cases}
$$

where $\tilde{a}$ is adjacent to wa in $\operatorname{Odd}(a)$. 
We are now able to give a detailed description of the components of $J$ whenever $W_{J}$ is the finite continuation of a simple reflection.

Proposition 35. Suppose that $a \in J \subseteq \Pi$ with $W_{J}=\mathrm{FC}\left(r_{a}\right)$, and suppose that $K$ is a component of $J$. Then one of the following alternatives holds.

(a) $K=\{a\}=J \cap \operatorname{Odd}(a)$.

(b) $K=\{a, b\}$ is of type $C_{2}$, and $J \cap \operatorname{Odd}(a)=\{a\}$.

(c) $K=\{a\}$ or $K=\{b\}$, where $\{a, b\}=J \cap \operatorname{Odd}(a)$ is of type $A_{1}+A_{1}$.

(d) $K=\{b\} \nsubseteq \subseteq \operatorname{Odd}(a)$, and $b$ is a $C_{3}$-neighbour of $\operatorname{Odd}(a)$.

(e) $\operatorname{Odd}(a) \subseteq K$, and $K$ is a component of $\operatorname{EOdd}(a)$.

(f) $K \cap \operatorname{Odd}(a)=\emptyset$, and $K$ is a component of $\operatorname{EOdd}(a)$.

Proof. We consider first the case that $K \cap \operatorname{Odd}(a) \neq \emptyset$, and start by supposing that there exists a spherical $L \subseteq \Pi$ with $J \subseteq L$ and $K$ not a component of $L$.

Choose such an $L$, and let $L_{0}$ be the component of $L$ containing $a$. By Proposition 27, since $K$ is not a component of $L$ we must have $K \subseteq L_{0}$. So either $K=\{a\}$, in which case (a) above holds, or else $\{a\} \varsubsetneqq\{a\} \cup K \subseteq J \cap L_{0}$. Furthermore, $J \cap L_{0} \neq L_{0}$, since $K \neq L_{0}$. So if (a) does not hold then $\{a\} \neq J \cap L_{0} \neq L_{0}$, and so one of the alternatives (1), (2) or (3) of Proposition 27 must hold.

Suppose that alternative (2) holds, so that $K=\{a, b\}=J \cap L_{0}$ for some $b$, and $\{a, b\}$ is of type $C_{2}$. By Proposition 28 we see that each $c \in \operatorname{Odd}(a) \backslash\{a\}$ lies in a type $C$ spherical subset $L^{\prime}$ of $\Pi$ containing $\{a, b\}$. Since $J \cap L^{\prime}=\{a, b\}$ (by Proposition 27) it follows that $c \notin J$. So $J \cap \operatorname{Odd}(a)=\{a\}$, and (b) above is satisfied.

Suppose that alternative (3) of Proposition 27 holds, so that $J \cap L_{0}=\{a, b\}$ is of type $A_{1}+A_{1}$, and $b \in \operatorname{Odd}(a)$. Proposition 29 immediately yields that $J \cap \operatorname{Odd}(a)=$ $\{a, b\}$, and so (c) above is satisfied.

Suppose that alternative (1) of Proposition 27 holds, so that $L_{0}=\{a, c, b\}$ with $m_{a c}=3$ and $m_{c b}=4$, and $J \cap L_{0}=\{a, b\}$. By Lemma 30 we know that $b \notin \operatorname{Odd}(a)$, and since we have assumed that $K \cap \operatorname{Odd}(a) \neq \emptyset$, it follows that $K=\{a\}=$ $J \cap \operatorname{Odd}(a)$. So (a) holds.

We have now dealt with all cases that arise if there is a spherical $L \subseteq \Pi$ with $J \subseteq L$ and $K$ not a component of $L$. So assume that $K$ is a component of every spherical $L$ containing $J$. We show that in this case $\operatorname{Odd}(a) \subseteq K$, and $K$ is a component of $\operatorname{EOdd}(a)$; that is, (e) above holds.

To show that $\operatorname{Odd}(a) \subseteq K$ it is clearly sufficient to show that if $a^{\prime} \in K \cap \operatorname{Odd}(a)$ and $b$ is adjacent to $a^{\prime}$ in $\operatorname{Odd}(a)$ then $b \in K$. Note that since $a^{\prime} \in \operatorname{Odd}(a)$ there exists $w \in W$ with $a^{\prime}=w a$, and since Proposition 25 yields that $w \in N_{W}\left(W_{J}\right)$ it follows that $\mathrm{FC}\left(r_{a^{\prime}}\right)=W_{J}$. Now the assumption that $b$ and $a^{\prime}$ are adjacent in $\operatorname{Odd}(a)$ implies that $\left\{a^{\prime}, b\right\}$ is spherical, and therefore $J \cup\{b\}$ is spherical. But $K$ is a component of 
every spherical subset of $\Pi$ containing $J$; so it is a component of $J \cup\{b\}$. But $a^{\prime} \in K$ and $b$ is adjacent to $a^{\prime}$; so $b \in K$, as required.

Since $K \subseteq J \subseteq \operatorname{EOdd}(a)$ and $K$ is connected, saying that $K$ is a component of $\operatorname{EOdd}(a)$ is equivalent to saying that $m_{b c}=2$ whenever $b \in K$ and $c \in \operatorname{EOdd}(a) \backslash K$. So suppose that $c \in \operatorname{EOdd}(a) \backslash K$. Then there exists an $a^{\prime} \in \operatorname{Odd}(a)$ such that $m_{a^{\prime} c}$ is even. Thus $\left\{a^{\prime}, c\right\}$ is spherical, and as above it follows that $J \cup\{c\}$ is spherical. So $K$ must be a component of $J \cup\{c\}$, and since $c \notin K$ it follows that $m_{b c}=2$ for all $b \in K$, as required.

It remains to consider the case that $K \cap \operatorname{Odd}(a)=\emptyset$; we must show that either (f) or (d) holds. We start by supposing that there exists a spherical $L \subseteq \Pi$ and a $w \in W$ with $w J \subseteq L$ and $w K$ not a component of $L$.

Choose such $L$ and $w$, and let $L_{0}$ be the component of $L$ containing $w a$. By Proposition 27, since $w K$ is not a component of $L$ we must have $w K \subseteq L_{0}$. Now $w J \cap L_{0} \neq L_{0}$ since $w K \neq L_{0}$, and $\{w a\} \neq w J \cap L_{0}$ since $w a \notin w K$. So one of the alternatives (1), (2) or (3) of Proposition 27 must hold. Alternative (3) can be ruled out, since in that case $w J \cap L_{0} \subseteq \operatorname{Odd}(w a)$, which is impossible since $K \cap \operatorname{Odd}(a)=\emptyset$. If alternative (2) holds then $w K=w J \cap L_{0}$ contains $w a$ and is of type $C_{2}$, whence $K$ contains $a$ and is of type $C_{2}$, and (b) is satisfied. If alternative (1) holds then since $w K \neq\{w a\}$ it follows from Proposition 32 that $w K=\{b\}$, with $b$ a $C_{3}$-neighbour of $\operatorname{Odd}(a)$. Since $w a$ is not adjacent to $b$, it follows from Lemma 34 that $w^{-1} b=b$, unless $a$ is adjacent to $b$, in which case $w^{-1} b=b+\sqrt{2} a+\sqrt{2} \tilde{a}$ for some $\tilde{a}$ in $\operatorname{Odd}(a)$. But this latter case cannot occur, since $w^{-1} b \in K \subseteq \Pi$. So $K=w K=\{b\}$, with $b$ a $C_{3}$-neighbour of $\operatorname{Odd}(a)$, and (d) holds.

Finally, suppose that $w K$ is a component of every spherical $L \subseteq \Pi$ such that $w J \subseteq L$ for some $w \in W$. For each $c \in \operatorname{EOdd}(a) \backslash K$ there is then a sequence $a=a_{0}, a_{1}, \ldots, a_{k}=c$ in $\Pi$ such that $m_{a_{i-1} a_{i}}$ finite for all $i \in\{1,2, \ldots, k\}$ and odd for all $i \in\{1,2, \ldots, k-1\}$. We shall show that, for every such sequence, $m_{b a_{i}}=2$ for all $b \in K$ and $i \in\{0,1, \ldots, k\}$; in particular, this will show that $m_{b c}=2$ whenever $b \in K$ and $c \in \operatorname{EOdd}(a) \backslash K$, enabling us to conclude that $K$ is a component of $\operatorname{EOdd}(a)$.

The case $k=0$ is clear, since $a \in J \backslash K$ and $K$ is a component of $J$. Proceeding by induction, we may assume that $k>0$ and $m_{b a_{i}}=2$ for all $i \in\{1,2, \ldots, k-1\}$ and all $b \in K$. We see that the element $u=v\left[a_{k-1},\left\{a_{k-2}\right\}\right] v\left[a_{k-2},\left\{a_{k-3}\right\}\right] \ldots v\left[a_{1},\left\{a_{0}\right\}\right]$ centralizes $W_{K}$ and has the property that $u a=a_{k-1}$, since the labels in the path from $a$ to $a_{k-1}$ are all odd. The group $u^{-1} W_{\left\{a_{k-1}, a_{k}\right\}} u$ is finite and contains $u^{-1} r_{a_{k-1}} u=r_{a}$, and so there is a maximal finite subgroup $G$ of $W$ containing this group and also containing $W_{J}$.

Note that $W_{J} \cup\left\{u^{-1} r_{c} u\right\} \subseteq G=w^{-1} W_{L} w$, for some $w \in W$ and spherical $L \subseteq \Pi$, the element $u$ being in the centralizer of $W_{K}$. We may choose $w$ to be the minimal length element of $W_{L} w=W_{L} w W_{J}$, and it then follows from Lemma 15 that $w J \subseteq L$. Hence $w K$ is a component of $L$. Furthermore, since $w u^{-1} r_{c} u w^{-1} \in W_{L}$ 
we see that the root $w u^{-1} c$ is in $\Phi_{L}$ and not in $\Phi_{w K}=w u^{-1} \Phi_{K}$ (since $c \notin \Phi_{K}$ ). So $w u^{-1} c \cdot w u^{-1} b=0$ for all $b \in K$. So $c \cdot b=0$, or (equivalently) $m_{b c}=2$ for all $b \in K$, as required.

To complement the results we have obtained so far, our next task is to find conditions that ensure that a visible subgroup $W_{K}$ is contained in $\mathrm{FC}\left(r_{a}\right)$.

Lemma 36. Let $a \in \Pi$ and $K$ a component of $\operatorname{EOdd}(a)$ such that $W_{K}$ is finite. Then $W_{K} \subseteq \mathrm{FC}\left(r_{a}\right)$.

Proof. Let $F$ be a maximal finite subgroup of $W$ with $r_{a} \in F$, and choose $w \in W$ such that $w F w^{-1}=W_{L}$ for some $L \subseteq \Pi$. We may replace $w$ by the minimal length element in the double coset $W_{L} w W_{\{a\}}$, since this does not affect the condition $w F w^{-1}=$ $W_{L}$. So we have that $w^{-1} L \subseteq \Phi^{+}$, and, moreover, $r_{a} \in w^{-1} L w \cap W_{\{a\}}=W_{w^{-1} L \cap\{a\}}$ by Lemma 15. So $w a \in L \subseteq \Pi$, and by Lemma 12 we see that $w$ is a product of factors of the form $v[d,\{c\}]$, with $c, d \in \operatorname{EOdd}(a)$. Since $K$ is a component of $\operatorname{EOdd}(a)$ it follows that each $v[d,\{c\}]$ normalizes $W_{K}$, and therefore $w$ normalizes $W_{K}$. Moreover, since $w a \in L$ and $L$ is spherical, it follows that $L \subseteq \operatorname{EOdd}(w a)=\operatorname{EOdd}(a)$. So $W_{L}$ normalizes $W_{K}$. But $W_{K}$ is finite, by hypothesis, and $W_{L}$ is a maximal finite subgroup of $W$. So $W_{K} \subseteq W_{L}$, and $W_{K}=w^{-1} W_{K} w \subseteq w^{-1} W_{L} w=F$. Thus $W_{K}$ is contained in all maximal finite subgroups of $W$ containing $r_{a}$, as required.

Lemma 37. Let $a \in \Pi$ and let $b$ be $a C_{3}$-neighbour of $\operatorname{Odd}(a)$. If $a$ and $b$ are not adjacent in $\Pi$ then $r_{b} \in \mathrm{FC}\left(r_{a}\right)$.

Proof. Let $F$ be a maximal finite subgroup of $W$ with $r_{a} \in F$. As in the proof of Lemma 36 there exist a $w \in W$ and a maximal spherical $L \subseteq \Pi$ with $w a=a^{\prime} \in L$ and $F=w^{-1} W_{L} w$. Since $L$ is spherical, $L \subseteq \operatorname{EOdd}(a)$.

Suppose first that $a^{\prime}$ is not adjacent to $b$. Then $m_{c a^{\prime}}=\infty$ for every $c \in \operatorname{Odd}(a)$ that is adjacent to $b$, and since $a^{\prime} \in L$ it follows that no such $c$ is in $L$. Thus $m_{b e}=2$ for all $e \in L \cap \operatorname{Odd}(a)$. But since also $m_{b e}=2$ for all $e \in \operatorname{EOdd}(a) \backslash(\operatorname{Odd}(a) \cup\{b\})$, it follows that $m_{b e}=2$ for all $e \in L \backslash\{b\}$. Thus $\{b\}$ is a component of $L \cup\{b\}$, and since $L$ is spherical it follows that $L \cup\{b\}$ is spherical. Maximality of $L$ tells us that $b \in L$. Moreover, Lemma 34 gives $w b=b$, and so $r_{b}=w^{-1} r_{b} w \in w^{-1} W_{L} w=F$.

On the other hand, suppose that $a^{\prime}$ is adjacent to $b$. In this case Lemma 34 gives $w b=b+\sqrt{2} a^{\prime}+\sqrt{2} \tilde{a}$, where $\tilde{a}$ is the unique neighbour of $a^{\prime}$ in $\operatorname{Odd}(a)$. Furthermore, since $m_{a^{\prime} e} \in\{2, \infty\}$ for all $e \in \Pi \backslash\left\{\tilde{a}, a^{\prime}, b\right\}$, we see that $m_{a^{\prime} e}=2$ for all $e \in L \backslash\left\{\tilde{a}, a^{\prime}, b\right\}$ (since $L$ is spherical). But the definition of a $C_{3}$-vertex also requires that $m_{\tilde{a} e}=m_{b e}=2$ whenever $m_{a^{\prime} e}=2$; so it follows that $\left\{\tilde{a}, a^{\prime}, b\right\}$ is a component of $L \cup\left\{\tilde{a}, a^{\prime}, b\right\}$, which is therefore spherical since $L$ and $\left\{\tilde{a}, a^{\prime}, b\right\}$ are both spherical. Maximality of $L$ tells us that $\left\{\tilde{a}, a^{\prime}, b\right\} \subseteq L$; so $w b=b+\sqrt{2} a^{\prime}+\sqrt{2} \tilde{a} \in \Phi_{L}$, and $r_{b}=w^{-1} r_{w b} w \in w^{-1} W_{L} w=F$. 
So $r_{b} \in F$ in all cases, and so $r_{b}$ is contained in all maximal finite subgroups of $W$ containing $r_{a}$, as required.

We now prove the converse to Proposition 28.

Proposition 38. Let $a \in \Pi$ and $b \in \Pi \backslash \operatorname{Odd}(a)$, and suppose that $(a, b)$ is a focus of $\operatorname{Odd}(a)$ in $\Pi$. Then $\mathrm{FC}\left(r_{a}\right)=W_{J}$, where $J$ is the union of $\{a, b\}$ and the spherical components of $\operatorname{EOdd}(a)$. Moreover, $\mathrm{FC}\left(r_{a^{\prime}}\right)$ is not visible for any $a^{\prime} \in \operatorname{Odd}(a) \backslash\{a\}$.

Proof. For each $c \in \operatorname{Odd}(a)$ let $X(c)=b+\sqrt{2} \sum_{i=1}^{m} c_{i}$ and $Y(c)=b+\sqrt{2} \sum_{i=1}^{m-1} c_{i}$, where $c_{1}=a, c_{2}, \ldots, c_{m}=c$ is the unique path from $a$ to $c$ in $\operatorname{Odd}(a)$, noting that $X(c)$ and $Y(c)$ are roots in $\Phi_{C[b . . c]}$. We remark, for later use, that $X(c)$ and $Y(c)$ are fixed by the reflections $r_{b}, r_{c_{1}}, \ldots, r_{c_{m-2}}$.

Let $F=w^{-1} W_{L} w$ be a maximal finite subgroup of $W$ containing $r_{a}$, with $L \subseteq \Pi$ and $w$ of minimal length in $W_{L} w W_{a}$. Then $w a=a^{\prime} \in L$, by Lemma 15. Put $L_{0}=L \cap \operatorname{Odd}(a)$.

Choose $c \in L_{0}$ with $C[b . . c]$ of maximal cardinality. If $d \in L_{0}$ then $m_{c d} \neq \infty$ (since $L_{0}$ is spherical), whence $d \in C[b . . c]$ by condition (3) of Definition 5. So $L_{0} \subseteq C[b . . c]$. Now if $e \in L \backslash L_{0}$ is arbitrary then $e \notin \operatorname{Odd}(a)$ (since $e \notin L \cap \operatorname{Odd}(a)$ ) and $m_{c e}<\infty$ (since $c, e \in L$ and $L$ is spherical). By condition (4) of Definition 5 it follows that $m_{d e}=2$ for all $d \in C[b . . c]$. Since this holds for all $e \in L \backslash L_{0}$, and $C[b . . c]$ and $L \backslash L_{0}$ are both spherical, it follows that $C[b . . c] \cup\left(L \backslash L_{0}\right)$ is spherical. But this set contains $L$ (since $L_{0} \subseteq C[b . . c]$ ) and since $L$ is a maximal spherical subset of $\Pi$ we conclude that $L=C[b . . c] \cup\left(L \backslash L_{0}\right)$.

By Proposition 12 and Lemma 9 there exist simple roots $e_{1}, e_{2}, \ldots, e_{k}$ and $d_{1}=a, d_{2}, \ldots, d_{k+1}=a^{\prime} \in \operatorname{Odd}(a)$ with $w=v\left[e_{k},\left\{d_{k}\right\}\right] \ldots v\left[e_{2},\left\{d_{2}\right\}\right] v\left[e_{1},\left\{d_{1}\right\}\right]$ and $v\left[e_{i},\left\{d_{i}\right\}\right] d_{i}=d_{i+1}$ for all $i \in\{1,2, \ldots, k\}$. Moreover, $m_{e_{i} d_{i}}<\infty$ for all $i$. Let $w_{0}=1$ and $w_{i}=v\left[e_{i},\left\{d_{i}\right\}\right] w_{i-1}$; we will show that

$$
\begin{aligned}
\left\{w_{i} b,\right. & \left.-w_{i} b, w_{i}(b+\sqrt{2} a),-w_{i}(b+\sqrt{2} a)\right\} \\
= & \left\{X\left(d_{i+1}\right),-X\left(d_{i+1}\right), Y\left(d_{i+1}\right),-Y\left(d_{i+1}\right)\right\}
\end{aligned}
$$

for all $i \in\{0,1, \ldots, k\}$. The case $i=0$ is trivial.

Proceeding inductively, suppose that $i>1$ and

$$
\left\{ \pm w_{i-1} b, \pm w_{i-1}(b+\sqrt{2} a)\right\}=\left\{ \pm X\left(d_{i}\right), \pm Y\left(d_{i}\right)\right\} .
$$

It will be sufficient to show that $v\left[e_{i},\left\{d_{i}\right\}\right] X\left(d_{i}\right)$ and $v\left[e_{i},\left\{d_{i}\right\}\right] Y\left(d_{i}\right)$ both lie in the set $\left\{ \pm X\left(d_{i+1}\right), \pm Y\left(d_{i+1}\right)\right\}$.

Suppose first that $d_{i}=a$. Then $X\left(d_{i}\right)=b+\sqrt{2} a$ and $Y\left(d_{i}\right)=b$. If $e_{i} \notin$ $\operatorname{Odd}(a)$ then $m_{e_{i} d_{i}}$ is even, and $d_{i+1}=d_{i}=a$. Furthermore, by condition (4) of Definition 5 we have either $\left\{e_{i}, d_{i}\right\}=\{b, a\}$ or $m_{e_{i} b}=m_{e_{i} a}=2$. In the former case 
$v\left[e_{i},\left\{d_{i}\right\}\right]=v[b,\{a\}]=r_{b} r_{a} r_{b}$, giving $v\left[e_{i},\left\{d_{i}\right\}\right] b=-b-\sqrt{2} a=-X(a)$ and $v\left[e_{i},\left\{d_{i}\right\}\right](b+\sqrt{2} a)=-b=-Y(a)$; in the latter case $v\left[e_{i},\left\{d_{i}\right\}\right]=v\left[e_{i}\{a\}\right]=r_{e_{i}}$, giving $v\left[e_{i},\left\{d_{i}\right\}\right] b=b=Y(a)$ and $v\left[e_{i},\left\{d_{i}\right\}\right](b+\sqrt{2} a)=b+\sqrt{2} a=X(a)$. If $e_{i} \in$ $\operatorname{Odd}(a)$ then $a \in C\left[b . . e_{i}\right]$, and by condition (2) of Definition 5 we have $m_{e_{i} b}=2$ and either $m_{e_{i} a}=2$ or $m_{e_{i} a}=3$. If $m_{e_{i} a}=2$ then $d_{i+1}=d_{i}=a$, while if $m_{e_{i} a}=3$ then $d_{i+1}=e_{i}$. Furthermore, in former case we find that $v\left[e_{i},\left\{d_{i}\right\}\right] b=r_{e_{i}} b=b=Y(a)$ and $v\left[e_{i},\left\{d_{i}\right\}\right](b+\sqrt{2} a)=b+\sqrt{2} a=X(a)$, while in the latter case we find that $v\left[e_{i},\left\{d_{i}\right\}\right] b=r_{a} r_{e_{1}} b=b+\sqrt{2} a=Y\left(e_{i}\right)$ and $v\left[e_{i},\left\{d_{i}\right\}\right]\left(b+\sqrt{2} a+\sqrt{2} e_{i}\right)=X\left(e_{i}\right)$.

Now suppose that $d_{i} \neq a$. If $e_{i} \notin \operatorname{Odd}(a) \cup\{b\}$ then $m_{e_{i} d_{i}}=2$ and $d_{i+1}=d_{i}$. Moreover, $m_{e_{i} d}=2$ for all $d \in C\left[b . . d_{i}\right]$, and so $v\left[e_{i},\left\{d_{i}\right\}\right]=r_{e_{i}}$ fixes all the roots in $\Phi_{C\left[b . . d_{i}\right]}$, including $X\left(d_{i}\right)=X\left(d_{i+1}\right)$ and $Y\left(d_{i}\right)=Y\left(d_{i+1}\right)$. If $e_{i} \in \operatorname{Odd}(a) \cup\{b\}$ and $\left\{e_{i}, d_{i}\right\}$ is not an edge of $\operatorname{Odd}(a)$ then we again have $d_{i+1}=d_{i}$ and $v\left[e_{i},\left\{d_{i}\right\}\right]=r_{e_{i}}$. By condition (3) of Definition 5 we either have $d_{i} \in C\left[b \ldots e_{i}\right]$ or $e_{i} \in C\left[b . . d_{i}\right]$. In the former case we have $m_{e_{i} d}=2$ for all $d \in C\left[b . . d_{i}\right]$, and as above we see that $r_{e_{i}}$ fixes $X\left(d_{i}\right)$ and $Y\left(d_{i}\right)$. In the latter case the remark made at the start of the proof implies that it is still true that $r_{e_{i}}$ fixes $X\left(d_{i}\right)$ and $Y\left(d_{i}\right)$. So we have shown that when $m_{e_{i} d_{i}}=2$ it is true that $v\left[e_{i},\left\{d_{i}\right\}\right] X\left(d_{i}\right)$ and $v\left[e_{i},\left\{d_{i}\right\}\right] Y\left(d_{i}\right)$ both lie in the set $\left\{ \pm X\left(d_{i+1}\right), \pm Y\left(d_{i+1}\right)\right\}$, and it remains only to consider the case that $e_{i}$ and $d_{i}$ are adjacent in $\operatorname{Odd}(a)$. Note that in this case $d_{i+1}=e_{i}$.

Let $C\left[b . . d_{i}\right]=\left\{b, c_{1}, \ldots, c_{m}\right\}$ with $c_{1}=a$ and $c_{m}=d_{i}$, and suppose that $e_{i}=c_{m-1}$ is the vertex adjacent to $d_{i}$ in $C\left[b . . d_{i}\right]$. Then

$$
\begin{aligned}
v\left[e_{i},\left\{d_{i}\right\}\right] X\left(d_{i}\right) & =r_{c_{m}} r_{c_{m-1}}\left(b+\sqrt{2} \sum_{j=1}^{m} c_{j}\right) \\
& =r_{c_{m}}\left(b+\sqrt{2} \sum_{j=1}^{m} c_{j}\right)=b+\sqrt{2} \sum_{j=1}^{m-1} c_{j}=X\left(e_{i}\right),
\end{aligned}
$$

and similarly

$$
\begin{aligned}
v\left[e_{i},\left\{d_{i}\right\}\right] Y\left(d_{i}\right) & =r_{c_{m}} r_{c_{m-1}}\left(b+\sqrt{2} \sum_{j=1}^{m-1} c_{j}\right) \\
& =r_{c_{m}}\left(b+\sqrt{2} \sum_{j=1}^{m-2} c_{j}\right)=b+\sqrt{2} \sum_{j=1}^{m-2} c_{j}=Y\left(e_{i}\right) .
\end{aligned}
$$

The alternative possibility is that $d_{i}$ is adjacent to $e_{i}$ in $C\left[b . . e_{i}\right]$. Exactly the same calculations show that $v\left[e_{i},\left\{d_{i}\right\}\right] X\left(d_{i}\right)=X\left(e_{i}\right)$ and $v\left[e_{i},\left\{d_{i}\right\}\right] Y\left(d_{i}\right)=Y\left(e_{i}\right)$ in this case also. 
The induction is now complete, and it follows in particular that $w b=w_{k} b$ is one of $\pm X\left(a^{\prime}\right)$ or $\pm Y\left(a^{\prime}\right)$. Hence

$$
w b \in \Phi_{C\left[b . . a^{\prime}\right]} \subseteq \Phi_{C[b . . c]} \subseteq \Phi_{L} .
$$

Thus $w r_{b} w^{-1} \in W_{L}$, and so $r_{b} \in w^{-1} W_{L} w=F$. Since $F$ was an arbitrary maximal finite subgroup of $W$ containing $r_{a}$, this shows that $r_{b} \in \mathrm{FC}\left(r_{a}\right)$.

Let $\widetilde{M}$ be the component of $\operatorname{EOdd}(a)$ containing $\operatorname{Odd}(a)$, and suppose, for a contradiction, that $\tilde{M}$ is spherical. Clearly $b \in \tilde{M}$, as $m_{b a}=4$, but $\widetilde{M}=\operatorname{Odd}(a) \cup\{b\}$ is not permitted, in view of condition (5) of Definition 5. So $\widetilde{M} \backslash(\operatorname{Odd}(a) \cup\{b\}) \neq \emptyset$. But for $e \in \widetilde{M} \backslash(\operatorname{Odd}(a) \cup\{b\})$ and $c \in \operatorname{Odd}(a)$ we have $m_{c e} \neq \infty$, since $\widetilde{M}$ is spherical, and by condition (4) of Definition 5 it follows that $m_{b e}=m_{c e}=2$ for all $c \in \operatorname{Odd}(a)$. This contradicts the fact that $\tilde{M}$ is connected.

Now suppose that $a^{\prime} \in \operatorname{Odd}(a)$ is such that $\operatorname{FC}\left(r_{a^{\prime}}\right)=W_{J}$ for some $J \subseteq \Pi$, and let $J_{0}$ be the component of $J$ containing $a^{\prime}$. Since $J_{0} \neq \widetilde{M}$ it follows from Proposition 35 that $J_{0}$ has rank at most 2. Now since there exists $w \in W_{C\left[b . . a^{\prime}\right]}$ such that $w a=-a^{\prime}$ and $w b=X\left(a^{\prime}\right)$, and since $r_{b} \in \mathrm{FC}\left(r_{a}\right)$, it follows that $r_{w b}=w r_{b} w^{-1} \in \mathrm{FC}\left(w r_{a} w^{-1}\right)=\mathrm{FC}\left(r_{a^{\prime}}\right)$. Thus $X\left(a^{\prime}\right) \in \Phi_{J}$, and so $C\left[b . . a^{\prime}\right] \subseteq J$. Since $J_{0}$ has rank at most 2, this means that $a^{\prime}=a$ and $J_{0}=\{a, b\}$.

It remains to prove that $J$ is the union of $J_{0}$ and the spherical components of $\operatorname{EOdd}(a)$. By Lemma 36 we know that all these components are contained in $J$. But if $K$ is any other component of $J$ such that $K \cap \operatorname{Odd}(a)=\emptyset$, then by Proposition 35 we see that $K=\left\{b^{\prime}\right\}$, with $b^{\prime}$ a $C_{3}$-neighbour of $\operatorname{Odd}(a)$. Since $b$ is the only element of $\Pi$ such that $m_{b c} \in\{2,4\}$ for all $c \in \operatorname{Odd}(a)$, we must have $b^{\prime}=b$, contradicting the fact that the component of $J$ containing $b$ is $J_{0}=\{a, b\}$.

Next, we have the converse to Proposition 29.

Proposition 39. Let $a \in \Pi$ and suppose that there exists $a b \in \operatorname{Odd}(a)$ such that $\{a, b\}$ is a half-focus of $\operatorname{Odd}(a)$ in $\Pi$. Suppose also that the vertices $\operatorname{Odd}(a) d o$ not comprise a spherical subset of $\Pi$. Then $\mathrm{FC}\left(r_{a}\right)=W_{J}$, where $J$ is the union of $\{a, b\}$ and the spherical components of $\operatorname{EOdd}(a)$. Moreover, $\mathrm{FC}\left(r_{a^{\prime}}\right)$ is not visible for any $a^{\prime} \in \operatorname{Odd}(a) \backslash\{a, b\}$.

Proof. For each $c \in \operatorname{Odd}(a) \backslash\{a, b\}$, define

$$
X(c)=b+a+c+2 \sum_{i=2}^{m-1} c_{i}
$$

where $c_{1}=a, c_{2}, \ldots, c_{m}=c$ is the unique path from $a$ to $c$ in $\operatorname{Odd}(a) \backslash\{b\}$. Then $X(c)$ is a root in $\Phi_{D[a, b . c]}$ and is fixed by the reflections $r_{b}, r_{c_{1}}, \ldots, r_{c_{m-2}}$ and $r_{c_{m}}$. Define also $X(a)=b$ and $X(b)=a$. 
Let $F=w^{-1} W_{L} w$ be a maximal finite subgroup of $W$ containing $r_{a}$, with $L \subseteq \Pi$ and $w$ of minimal length in $W_{L} w W_{a}$. Then $w a=a^{\prime} \in L$, by Lemma 15. Put $L_{0}=L \cap \operatorname{Odd}(a)$.

Choose $c \in L_{0}$ with $D[a, b . . c]$ of maximal cardinality. If $d \in L_{0}$ then $m_{c d} \neq \infty$ (since $L_{0}$ is spherical), whence $d \in D[a, b . . c]$ by condition (4) of Definition 6 . So $L_{0} \subseteq D[a, b . . c]$. Now if $e \in L \backslash L_{0}$ is arbitrary then $e \notin \operatorname{Odd}(a)$ (since $e \notin L \cap \operatorname{Odd}(a)$ ) and $m_{c e}<\infty$ (since $c, e \in L$ and $L$ is spherical). By condition (5) of Definition 6 it follows that $m_{d e}=2$ for all $d \in D[a, b . . c]$. Since this holds for all $e \in L \backslash L_{0}$, and $D[a, b . . c]$ and $L \backslash L_{0}$ are both spherical, it follows that $D[a, b . . c] \cup\left(L \backslash L_{0}\right)$ is spherical. But this set contains $L$ (since $L_{0} \subseteq D[a, b . . c]$ ) and since $L$ is a maximal spherical subset of $\Pi$ we conclude that $L=D[a, b . . c] \cup\left(L \backslash L_{0}\right)$.

By Proposition 12 and Lemma 9 there exist simple roots $e_{1}, e_{2}, \ldots, e_{k}$ and $d_{1}=$ $a, d_{2}, \ldots, d_{k+1}=a^{\prime} \in \operatorname{Odd}(a)$ with $w=v\left[e_{k},\left\{d_{k}\right\}\right] \ldots v\left[e_{2},\left\{d_{2}\right\}\right] v\left[e_{1},\left\{d_{1}\right\}\right]$ and $v\left[e_{i},\left\{d_{i}\right\}\right] d_{i}=d_{i+1}$ for all $i \in\{1,2, \ldots, k\}$. Furthermore, we have $m_{e_{i} d_{i}}<\infty$ for all $i$. Let $w_{0}=1$, and $w_{i}=v\left[e_{i},\left\{d_{i}\right\}\right] w_{i-1}$ for $i \geqslant 1$. We will show that

$$
\left\{w_{i} b,-w_{i} b\right\}=\left\{X\left(d_{i+1}\right),-X\left(d_{i+1}\right)\right\}
$$

for all $i \in\{0,1, \ldots, k\}$.

The case $i=0$ is trivial. Proceeding inductively, suppose that $i>1$ and $w_{i-1} b=$ $\pm X\left(d_{i}\right)$. It will be sufficient to show that $v\left[e_{i},\left\{d_{i}\right\}\right] X\left(d_{i}\right)= \pm X\left(d_{i+1}\right)$.

Suppose first that $d_{i}=a$, so that $X\left(d_{i}\right)=b$. If $e_{i} \neq b$ then $m_{e_{i} b}=m_{e_{i} a} \in\{2,3\}$, since $m_{e_{i} a}=m_{e_{i} d_{i}} \neq \infty$. We also have $m_{e_{i} a}=2$ if $e_{i}=b$. In the case $m_{e_{i} a}=3$ we have $v\left[e_{i},\left\{d_{i}\right\}\right]=r_{a} r_{e_{i}}$, and $d_{i+1}=r_{a} r_{e_{i}} a=e_{i}$. Furthermore,

$$
v\left[e_{i},\left\{d_{i}\right\}\right] X\left(d_{i}\right)=r_{a} r_{e_{i}} b=a+b+e_{i}=X\left(e_{i}\right)=X\left(d_{i+1}\right),
$$

as required. In the case $m_{e_{i} a}=2$ we have $v\left[e_{i},\left\{d_{i}\right\}\right]=r_{e_{i}}$, giving $d_{i+1}=r_{e_{i}} a=a$, and

$$
v\left[e_{i},\left\{d_{i}\right\}\right] X\left(d_{i}\right)=r_{e_{i}} b= \pm b= \pm X\left(d_{i+1}\right),
$$

since either $e_{i}=b$ or $m_{e_{i} b}=2$.

The case $d_{i}=b$ is the same as the case $d_{i}=a$ with $a$ and $b$ interchanged; so suppose that $d_{i} \notin\{a, b\}$. If $e_{i} \notin \operatorname{Odd}(a)$ then $m_{e_{i} d_{i}}=2$ and $d_{i+1}=d_{i}$. Moreover, $m_{e_{i} d}=2$ for all $d \in D\left[a, b . . d_{i}\right]$, and so $v\left[e_{i},\left\{d_{i}\right\}\right]=r_{e_{i}}$ fixes all the roots in $\Phi_{D\left[a, b . d_{i}\right]}$, including $X\left(d_{i}\right)=X\left(d_{i+1}\right)$. If $e_{i} \in \operatorname{Odd}(a)$ and $\left\{e_{i}, d_{i}\right\}$ is not an edge of $\operatorname{Odd}(a)$ then we again have $d_{i+1}=d_{i}$ and $v\left[e_{i},\left\{d_{i}\right\}\right]=r_{e_{i}}$. By condition (4) of Definition 6 we either have $d_{i} \in D\left[a, b . . e_{i}\right]$ or $e_{i} \in D\left[a, b . . d_{i}\right]$. In the former case we have $m_{e_{i} d}=2$ for all $d \in C\left[b . . d_{i}\right]$, and as above we see that $r_{e_{i}}$ fixes $X\left(d_{i}\right)$. In the latter case it is still true that $r_{e_{i}}$ fixes $X\left(d_{i}\right)$, since the only simple reflection of $D\left[a, b . . d_{i}\right]$ that does not fix $X\left(d_{i}\right)$ is the one corresponding to the vertex adjacent to $d_{i}$. So we have shown that when $m_{e_{i} d_{i}}=2$ it is true that $v\left[e_{i},\left\{d_{i}\right\}\right] X\left(d_{i}\right)$ and 
$v\left[e_{i},\left\{d_{i}\right\}\right] Y\left(d_{i}\right)$ both lie in the set $\left\{ \pm X\left(d_{i+1}\right), \pm Y\left(d_{i+1}\right)\right\}$, and it remains to consider the case that $e_{i}$ and $d_{i}$ are adjacent in $\operatorname{Odd}(a)$. Note that in this case $d_{i+1}=e_{i}$.

Let $D\left[a, b . . d_{i}\right]=\left\{b, c_{1}, \ldots, c_{m}\right\}$ with $c_{1}=a$ and $c_{m}=d_{i}$. Suppose first that $m>2$, and suppose that $e_{i}=c_{m-1}$ is the vertex adjacent to $d_{i}$ in $D\left[a, b . . d_{i}\right]$. Then

$$
\begin{aligned}
v\left[e_{i},\left\{d_{i}\right\}\right] X\left(d_{i}\right) & =r_{c_{m}} r_{c_{m-1}}\left(b+a+c_{m}+2 \sum_{j=2}^{m-1} c_{j}\right) \\
& =r_{c_{m}}\left(b+a+c_{m}+c_{m-1}+2 \sum_{j=1}^{m-2} c_{j}\right) \\
& =b+a+c_{m-1}+2 \sum_{j=1}^{m-2} c_{j}=X\left(e_{i}\right) .
\end{aligned}
$$

If $m=2$ and $e_{i}=b$ then

$$
v\left[e_{i},\left\{d_{i}\right\}\right] X\left(d_{i}\right)=r_{b} r_{d_{i}}\left(a+b+d_{i}\right)=a=X(b)=X\left(e_{i}\right),
$$

and if $e_{i}=a$ then similarly

$$
v\left[e_{i},\left\{d_{i}\right\}\right] X\left(d_{i}\right)=r_{a} r_{d_{i}}\left(a+b+d_{i}\right)=b=X(a)=X\left(e_{i}\right) .
$$

The alternative possibility is that $d_{i}=c_{m-1}$ is the vertex adjacent to $e_{i}=c_{m}$ in $D\left[a, b . . e_{i}\right]=\left\{b, c_{1}, \ldots, c_{m}\right\}$. We calculate that

$$
\begin{aligned}
v\left[e_{i},\left\{d_{i}\right\}\right] X\left(d_{i}\right) & =r_{c_{m-1}} r_{c_{m}}\left(b+a+c_{m-1}+2 \sum_{j=2}^{m-2} c_{j}\right) \\
& =r_{c_{m-1}}\left(b+a+c_{m}+c_{m-1}+2 \sum_{j=1}^{m-2} c_{j}\right) \\
& =b+a+c_{m}+2 \sum_{j=1}^{m-1} c_{j}=X\left(e_{i}\right),
\end{aligned}
$$

as required.

The induction is now complete, and it follows that $w b=w_{k} b= \pm X\left(a^{\prime}\right)$. Hence

$$
w b \in \Phi_{D\left[a, b . . a^{\prime}\right]} \subseteq \Phi_{D[a, b . c]} \subseteq \Phi_{L} .
$$

Thus $w r_{b} w^{-1} \in W_{L}$, and so $r_{b} \in w^{-1} W_{L} w=F$. Since $F$ was an arbitrary maximal finite subgroup of $W$ containing $r_{a}$, this shows that $r_{b} \in \mathrm{FC}\left(r_{a}\right)$.

Note that since $W$ has a graph automorphism that swaps $r_{a}$ and $r_{b}$ and fixes all the other simple reflections, it must also be true that $r_{a} \in \mathrm{FC}\left(r_{b}\right)$. 
Let $\widetilde{M}$ be the component of $\operatorname{EOdd}(a)$ containing $\operatorname{Odd}(a)$, and suppose, for a contradiction, that $\widetilde{M}$ is spherical. Note that $\widetilde{M} \neq \operatorname{Odd}(a)$, in view of condition (5) of Definition 5. So $\widetilde{M} \backslash \operatorname{Odd}(a) \neq \emptyset$. But for all $e \in \tilde{M} \backslash \operatorname{Odd}(a)$ and $c \in \operatorname{Odd}(a)$ we have $m_{c e} \neq \infty$, since $\widetilde{M}$ is spherical, and by conditions (1) and (5) of Definition 5 it follows that $m_{c e}=2$ for all $c \in \operatorname{Odd}(a)$. This contradicts the fact that $\widetilde{M}$ is connected.

Suppose that $a^{\prime} \in \operatorname{Odd}(a)$ is such that $\mathrm{FC}\left(r_{a^{\prime}}\right)=W_{J}$ for some $J \subseteq \Pi$, and let $J_{0}$ be the component of $J$ containing $a^{\prime}$. Since $J_{0} \neq \tilde{M}$ it follows from Proposition 35 that $J \cap \operatorname{Odd}(a)$ has rank at most 2. Now suppose, for a contradiction, that $a^{\prime} \notin\{a, b\}$. Since there exists an element $w \in W_{D\left[a, b . . a^{\prime}\right]}$ such that $w a=a^{\prime}$ and $w b=X\left(a^{\prime}\right)$, it follows that $r_{w b}=w r_{b} w^{-1} \in \mathrm{FC}\left(w r_{a} w^{-1}\right)=\mathrm{FC}\left(r_{a^{\prime}}\right)$. Thus $X\left(a^{\prime}\right) \in \Phi_{J}$, and so $D\left[a, b . . a^{\prime}\right] \subseteq J$, contradicting the fact that the rank of $J \cap \operatorname{Odd}(a)$ is at most 2. So we deduce that $a^{\prime}=b$ or $a^{\prime}=a$. Moreover, in either case we know that $\{a, b\} \subseteq J \cap \operatorname{Odd}(a)$, and since $J \cap \operatorname{Odd}(a)$ has rank at most 2 it follows that $J \cap \operatorname{Odd}(a)=\{a, b\}$.

By Lemma 36 we know that all spherical components of EOdd $(a)$ are components of $J$, and by Proposition 35 all other components of $J$ that intersect Odd $(a)$ trivially correspond to $C_{3}$-neighbours of $\operatorname{Odd}(a)$. But clearly the conditions of Definition 6 imply that $\operatorname{Odd}(a)$ has no $C_{3}$-neighbours. So we conclude that $J$ is the union of $\{a, b\}$ and the spherical components of $\operatorname{EOdd}(a)$, as required.

Proof of Theorem 7. Let $M$ be a connected component of $\Omega(\Pi)$, and write $\widetilde{M}$ for the component of $\mathrm{E}(M)$ containing $M$.

Suppose first that $\widetilde{M}$ is spherical, so that the conditions of Case A of Theorem 7 are satisfied, and let $a \in M$ be arbitrary. Observe that all $C_{3}$-neighbours of $M$ are contained in $\widetilde{M}$. Choose $a^{\prime} \in M$ such that $\operatorname{FC}\left(r_{a^{\prime}}\right)$ is visible, and let $\operatorname{FC}\left(r_{a^{\prime}}\right)=$ $W_{J}$. By Lemma 36 we know that $\tilde{M}$ is contained in $J$, and hence $a \in J$. So by Proposition 25 it follows that $\mathrm{FC}\left(r_{a}\right)=W_{J}$ also. By Proposition 35 the only possible components of $J$ apart from $J_{0}$ are the other spherical components of $\mathrm{E}(M)$, and by Lemma 36 all of these are indeed components of $J$. So $J$ consists of the spherical components of $\mathrm{E}(M)$, as required.

Now suppose that $\widetilde{M}$ is not spherical. If there exists a $b \in \Pi \backslash M$ such that $(a, b)$ is a focus of $M$ then it follows from Proposition 38 that $\mathrm{FC}\left(r_{a}\right)=W_{J}$, where $J$ is composed of $\{a, b\}$ and the spherical components of $\mathrm{E}(M)$, and $\mathrm{FC}\left(r_{a^{\prime}}\right)$ is not visible for any $a^{\prime} \in \operatorname{Odd}(a) \backslash\{a\}$. Similarly, if there exists a $b \in M$ such that $\{a, b\}$ is a half-focus of $M$, then it follows from Proposition 39 that $\operatorname{FC}\left(r_{a}\right)=\operatorname{FC}\left(r_{b}\right)=W_{J}$, where $J$ is composed of $\{a, b\}$ and the spherical components of $\mathrm{E}(M)$, and $\mathrm{FC}\left(r_{a^{\prime}}\right)$ is not visible for any $a^{\prime} \in \operatorname{Odd}(a) \backslash\{a, b\}$.

Finally, suppose that $\widetilde{M}$ is not spherical and $M$ does not have a focus or a half focus. Suppose that $a \in M$ is such that $\mathrm{FC}\left(r_{a}\right)=W_{J}$ for some $J \subseteq \Pi$, and let $K$ be the component of $J$ containing $a$. 
Suppose first that alternative (b) of Proposition 35 holds, so that $K=\{a, b\}$ is of type $C_{2}$, and $J \cap M=\{a\}$. Since $M$ does not have any focus in $\Pi$, it follows from Proposition 28 that $M \cup\{b\}$ is a spherical component of $\mathrm{E}(M)$. But the component of $\mathrm{E}(M)$ containing $M$ is $\tilde{M}$, which, by our assumptions, is not spherical. So this case does not arise.

Alternative (c) of Proposition 35 is similarly impossible, by Proposition 29, and alternative (e) is also incompatible with the assumption that $\widetilde{M}$ is not spherical. So we conclude that alternative (a) holds: $K=\{a\}=J \cap \operatorname{Odd}(a)$. Note that all spherical components of $\mathrm{E}(M)$ are components of $J$, and by Proposition 35 the only other possible components are the sets $\{b\}$ such that $b$ is a $C_{3}$-neighbour of $M$.

Suppose that $b$ is a $C_{3}$-neighbour of $M$ that is adjacent to $a$. Let $\tilde{a}$ be the unique neighbour of $a$ in $M$. By Lemma 37 we know that $r_{b} \in \mathrm{FC}\left(r_{\tilde{a}}\right)$, and so it follows that $\mathrm{FC}\left(r_{a}\right)=r_{\tilde{a}} r_{a} \mathrm{FC}\left(r_{\tilde{a}}\right) r_{a} r_{\tilde{a}}$ contains the reflection along the root $r_{\tilde{a}} r_{a} b=b+\sqrt{2} a+$ $\sqrt{2} \tilde{a}$. Since $\mathrm{FC}\left(r_{a}\right)=W_{J}$, it follows that both $b$ and $\tilde{a}$ are in $J$. But this contradicts the fact that the component of $J$ containing $a$ is just $\{a\}$.

This reasoning has shown that if $a \in M$ is adjacent in $\Pi$ to a $C_{3}$-neighbour of $M$ then $\mathrm{FC}\left(r_{a}\right)$ is not visible. On the other hand, we know that there is at least one $a \in M$ such that $\mathrm{FC}\left(r_{a}\right)$ is visible. So we may choose an $a \in M$ such that $\mathrm{FC}\left(r_{a}\right)=W_{J}$ for some $J \subseteq \Pi$. Since $a$ is not adjacent to any $C_{3}$-neighbour of $M$ it follows by Lemma 37 that all $C_{3}$-neighbours of $M$ are in $J$. So we conclude that $J=J^{\prime} \cup\{a\}$, where $J^{\prime}$ is the union of the spherical components of $\mathrm{E}(M)$ and the $C_{3}$-neighbours of $M$.

It remains to prove that if $a^{\prime}$ is any other element of $M$ that is not adjacent to any $C_{3}$-neighbour of $M$ then $\operatorname{FC}\left(r_{a^{\prime}}\right)=W_{J^{\prime} \cup\left\{a^{\prime}\right\}}$. Given such an $a^{\prime}$, since $a^{\prime}$ lies in $M=\operatorname{Odd}(a)$, we may choose $w \in W$ such that $w a=a^{\prime}$. By Proposition 12 we see that $w \in W_{\widetilde{M}}$, and so $w$ fixes all other components of $\mathrm{E}(M)$. And $w$ fixes all $C_{3}$-neighbours of $M$, by Lemma 34. So $w$ fixes $J^{\prime}$, and it follows that

$$
\mathrm{FC}\left(r_{a^{\prime}}\right)=w \mathrm{FC}\left(r_{a}\right) w^{-1}=w W_{J^{\prime} \cup\{a\}} w^{-1}=W_{w J^{\prime} \cup\{w a\}}=W_{J^{\prime} \cup\left\{a^{\prime}\right\}},
$$

as required. This completes the proof of Theorem 7 .

Proof of Theorem 1. Let $a \in \Pi$ and $M=\operatorname{Odd}(a)$. As $\Pi$ is 2-spherical it follows that $\Pi=E(M)$, and, as $\Pi$ is non-spherical, it follows that Case A of Theorem 7 does not hold for $M$. As there are no $\infty$-labels in the Coxeter graph of $\Pi$, Cases $C$ and D do not hold either. Hence we are in Case B. Since there are no $\infty$-labels in the Coxeter graph of $\Pi$, there are no $C_{3}$ neighbors of $M$. As $E(M)=\Pi$ is irreducible, there are no spherical components of $E(M)$. It follows now from Theorem 7 that there is an $a^{\prime} \in \operatorname{Odd}(a)$ such that $\mathrm{FC}\left(r_{a^{\prime}}\right)=\left\langle r_{a^{\prime}}\right\rangle$. As $r_{a}$ and $r_{a^{\prime}}$ are $W$-conjugate we have $\mathrm{FC}\left(r_{a}\right)=\left\langle r_{a}\right\rangle$ as well, and this completes the proof of Part a) of Theorem 1.

Let $S \subseteq W$ be such that $(W, S)$ is a Coxeter system. It follows from Part a) and Corollary 24 that $r_{a} \in S^{W}$ for each $a \in \Pi$, and hence $\left\{r_{a} \mid a \in \Pi\right\} \subseteq S^{W}$. As $\Pi$ 
is assumed to be non-spherical, irreducible and 2-spherical, it follows now from the main result of [5] that there is an element $w \in W$ such that $\left\{r_{a} \mid a \in \Pi\right\}=S^{w}$. This completes the proof of Part b) of Theorem 1. As Part c) is an immediate consequence of Part b) we are done.

\section{References}

[1] P. Bahls and M. Mihalik, Reflection Independence in even Coxeter Groups. Geom. Dedicata 110 (2005), 63-80. Zbl 1072.20043 MR 2136020

[2] N. Bourbaki, Groupes et algèbres de Lie, Chapitres 4, 5 et 6. Actualités Scientifiques et Industrielles 1337, Hermann, Paris 1968. Zbl 0186.33001 MR 0240238

[3] N. Brady, J. McCammond, B. Mühlherr and W. Neumann, Rigidity of Coxeter Groups and Artin Groups. Geom. Dedicata 94 (2002), 91-109. Zbl 1031.20035 MR 1950875

[4] B. Brink, On centralizers of reflections in Coxeter groups. Bull. London Math. Soc. 28 (1996), 465-470. Zbl 0852.20033 MR 1396145

[5] P. E. Caprace and B. Mühlherr, Reflection rigidity of 2-spherical Coxeter groups. Preprint 2003, 24pp, to appear in Proc. London Math. Soc..

[6] Roger W. Carter, Finite Groups of Lie Type: Conjugacy Classes and Complex Characters. J. Wiley \& Sons, 1985. Zbl 0567.20023 MR 0794307

[7] V. V. Deodhar, On the root system of a Coxeter group. Comm. Algebra 10 (1982), 611-630. Zbl 0491.20032 MR 0647210

[8] V. V. Deodhar, A note on subgroups generated by reflections in Coxeter groups. Arch. Math. (Basel) 53 (1989), 543-546. Zbl 0688.20028 MR 1023969

[9] M. Dyer, Reflection subgroups of Coxeter systems. J. Algebra 135 (1990), 57-73. Zbl 0712.20026 MR 1076077

[10] W. N. Franzsen and R. B. Howlett, Automorphisms of nearly finite Coxeter groups. Adv. Geom. 3 (2003), 301-338. Zbl 1044.20022 MR 1997410

[11] R. B. Howlett, Normalizers of parabolic subgroups of reflection groups. J. London Math. Soc. (2) 21 (1980), 62-80. Zbl 0427.20040 MR 0576184

[12] R. B. Howlett and B. Mühlherr, Isomorphisms of Coxeter groups which do not preserve reflections. Preprint 2004, 18pp.

[13] R. B. Howlett, P. J. Rowley and D. E. Taylor, On outer automorphism groups of Coxeter groups. Manuscripta Math. 93 (1997), 499-513. Zbl 0888.20023 MR 1465894

[14] James E. Humphreys, Reflection Groups and Coxeter Groups. Stud. in Adv. Math. 29, Cambridge University Press, Cambridge 1990. Zbl 0768.20016 MR 1066460

[15] B. Mühlherr, The isomorphism problem for Coxeter groups. Preprint 2005, 15pp, to appear in The Coxeter Legacy: Reflections and Projections, Fields Institute Communications.

[16] R. W. Richardson, Conjugacy classes of involutions in Coxeter groups. Bull. Austral. Math. Soc. 26 (1982), 1-15. Zbl 0531.20017 MR 0679916 
Received February 23, 2005

W. N. Franzsen, Australian Catholic University, 25A Barker Rd, Strathfield NSW 2135, Australia

E-mail: b.franzsen@mary.acu.edu.au

R. B. Howlett, School of Mathematics and Statistics, University of Sydney, NSW 2006, Australia

E-mail: R.Howlett@maths.usyd.edu.au

B. Mühlherr, Département de Mathématiques, ULB C.P. 216, Bd. du Triomphe, 1050 Bruxelles, Belgium

E-mail: bernhard.muhlher@ulb.ac.be 